\title{
Tactile-based Whole-body Compliance with Force Propagation for Mobile Manipulators
}

\author{
Quentin Leboutet, Student Member, IEEE, Emmanuel Dean, Member, IEEE, Florian Bergner, Student \\ Member, IEEE, and Gordon Cheng, Fellow, IEEE
}

\begin{abstract}
In this article, we propose a control method, providing mobile robots with whole-body compliance capabilities, in response to multi-contact physical interactions with their environment. The external forces applied to the robot, as well as their localization on its kinematic tree, are measured using a multimodal, self-configuring and self-calibrating artificial skin. We formulate a compliance control law in Cartesian space, as a set of quadratic optimization problems, solved in parallel for each limb involved in the interaction process. This specific formulation makes it possible to determine the torque commands required to generate the desired reactive behaviors, while taking the robot kinematic and dynamic constraints into account. When a given limb fails to produce the desired compliant behavior, the generalized force residual at the considered contact points is propagated to a parent limb in order to be adequately compensated. Hence, the robot's compliance range can be extended in a both robust and easily adjustable manner. The experiments performed on a dual-arm velocity-controlled mobile manipulator, show that our methodology is robust to nullspace interactions and robot physical constraints.
\end{abstract}

Index Terms-Physical Human-Robot Interaction, Active compliance, Quadratic programming, Artificial robot skin

\section{INTRODUCTION}

\section{A. Motivation}

A LTHOUGH initially intended for purely industrial or scientific purposes, robots are now about to democratize in many different sectors of society, such as home automation, medicine, education and even entertainment [1]-[3]. Being both less expensive and easier to control than their legged counterparts, wheeled mobile robots are in particular likely to be extensively used in a near future for most consumer or medical applications [4]. Operating in a human environment, such robots must be capable of coping with a wide variety of physical interactions - whether desired or accidental - in a both safe and predictable manner [5]. To that end, they have to be whole-body compliant. Compliance has been extensively studied over the past two decades [6]-[14]. Commonly exploited in the industry for mechanical assembly purposes [15], [16] or the realization of cooperative tasks [17], it is henceforth increasingly used in the context of rehabilitation [18] and assistive robotics [19]. However, its generalization to multi-contact interaction scenarios between humans and robots remains difficult for two main reasons. First of all, in this context, compliance is no longer restricted to the endeffectors. Instead it must be guaranteed for an arbitrary number

Institute for Cognitive Systems (ICS), Technical University of Munich, Faculty of Electrical Engineering and Computer Science, 45/II Karlstraße 80333 Munich, Germany. of contact points distributed throughout the entire body of the considered robot. Therefore, such a robot must either have a soft mechanical structure, associated with a proper actuation strategy, e.g., variable stiffness [10], [20], [21], or must otherwise be equipped with specific sensor modalities allowing suitable reactive behaviors to be emulated by its controller [4], [22]. On the other hand, the generation of compliant behaviors must be guaranteed despite the kinematic and dynamic limitations of the considered robot, such as joint limits, torque limits or singularities. In practice, wholebody compliance cannot be adequately implemented on a fixed manipulator since any physical interaction with the root of its kinematic chain or with a singular axis of motion, systematically results in the emergence of stiff configurations. However, its implementation remains relevant on a mobile robot, as in this case, the locomotion system can be used to propagate the external forces applied to non-compliant upper body configurations. Nevertheless, a good coordination between the locomotor limbs and the rest of the robot is still required in order to ensure that the generated behaviors are both consistent and potentially safe to an external user.

\section{B. Related Works}

The issue of whole-body compliance for wheeled mobile manipulators has already been widely discussed in the literature. The typical approach consists in using an admittancecontrolled mobile base [23] in combination with a passive or active-compliant upper body. The mobile base control interface is usually formulated in the operational space. This is, for example, the case in [24], where a force sensor placed at the end-effector of a single-arm mobile manipulator, provides direct control over the movements of its base. In [4], [25]-[29] the Cartesian position error between a compliant frame, linked to the robot's end-effector, and a fixed frame attached to its lower body is used by the mobile base controller to generate suitable reactive motions. Additional stability and posture objectives are included in [27] with excellent performances. Although this technique allows greater flexibility - given that the mobile base motion generation is no longer restricted to the end-effector - it may be vulnerable to singularities since it relies on the compliant motion of the upper-body with respect to a fixed point in the base frame. In [30][37], whole-body compliance is achieved by placing a forcetorque sensor either at the mechanical coupling between the robot's upper and lower body or directly on its mobile base. Simple and naturally robust to singularities, this strategy may nevertheless be sensitive to the effects of internal forces, 
resulting from upper-body's inertia. Generating motion on the mobile base with a compliant upper body can also be achieved at the joint level. This is, for example, the case in [38]-[40] where some specific joints of a compliant upper body are assigned with a particular degree of freedom on the mobile base. This method works well provided that the arms do not get too far from their nominal posture, in which case, base movements may become inconsistent. In [41] an approach based on generalized inverse dynamics exploits the feedback of dedicated joint torque sensors, for whole-body compliance implementation on a dual-arm wheeled mobile manipulator. Although intrinsically compliant, robots using joint-torque feedback are in general vulnerable to singularities, since a force applied to a singular posture will not generate any detectable torque. In practice, realizing compliant behaviors on a stiff robot is often challenging since major structural modifications are necessary to provide the considered platform with either adequate sensory modalities (e.g. force-torque sensors), or with specific actuators (e.g. variable stiffness). In this context, the concept of whole-body tactile feedback is a relevant alternative, since covering an entire robot with an artificial skin to get suitable force or application point measurements only requires limited structural changes. Widely investigated during the last two decades, several promising prototypes have since then been successfully developed and tested, such as [42]-[46]. The artificial skin developed in [45], [46] is of particular interest as it is capable of simultaneously measuring four different tactile modalities, namely pressure, pre-touch, temperature and vibrations. Mounted on a robot, this artificial skin can be used for active compliance realization as demonstrated in [22]. In our previous work [48], we took advantage of the pressure and pre-touch modalities in order to achieve whole-body compliance with force propagation on a dual-arm omnidirectional mobile manipulator. Using Quadratic Programming (QP), we proposed a method allowing to estimate the components of external forces which could not generate sufficient reactive torques on a considered limb of the robot, due to kinematic or dynamic constraints. Once identified, these forces were propagated onto the robot's mobile base, thereby generating proper reactive motions in the direction of the upper limb's constrained axis. Although we successfully extended the compliance range of our robot, this study also highlighted the limitations of the proposed method. The most fundamental of these limitations was the impossibility of precisely tuning the reactive motion dynamics, especially when the external forces were applied close to a singular axis of the upper limbs kinematic chain. As demonstrated in [14], this point is critical for safe human-robot interaction. Moreover, properly handling the robot physical constraints such as joint limits or torque limits was made difficult by the cell-wise formulation of the proposed algorithm. Finally, since one optimization problem had to be solved for each activated skin cell, the previous method was therefore rapidly limited by computational complexity issues, especially for the robots covered by large skin patches. With these in mind, we here reformulate the entire control framework, to have a generic and computationally efficient whole-body compliance algorithm for wheeled mobile robots.

\section{Our Approach}

In this work, we formulate the compliance control law in Cartesian space. The desired reactive dynamics, defined at the contact point level (with respect to the base frame of the considered robot limb ${ }^{1}$ ) is tracked by the controller. Quadratic Programming (QP) makes it possible to compute a set of proper control torques, allowing the robot to follow the desired reactive behavior as accurately as possible, while intrinsically complying with a set of predefined physical constraints. Besides being easily accessible to humans - thereby simplifying the tuning of our controller - a Cartesian formulation makes it possible to conveniently capture the dynamic residuals between the desired and the actual reactive motions of each robot limb. These residuals are then propagated onto a parent kinematic chain - in our case the mobile base of the robot - to be adequately compensated, thereby extending the whole robot compliance range. This paper is organized as follows: Section II provides a formal description of the whole-body contact point compliance problem. Section III describes the proposed approach for whole-body multiple-contact compliance with force propagation using tactile feedback and quadratic programming. The experiments, as well as their results, are presented and discussed in Section IV. Finally, Section V gives a brief conclusion and discusses the possible future works.

\section{Multi-Contact Cartesian Compliance}

\section{A. Preliminaries}

The dynamic model of a rigid serial manipulator with $n$ degrees of freedom, can be derived using the Euler-Lagrange's formalism [49], resulting in the following equation of motion:

$$
\mathbf{M}(\mathbf{q}) \ddot{\mathbf{q}}+\mathbf{C}(\mathbf{q}, \dot{\mathbf{q}}) \dot{\mathbf{q}}+\mathbf{g}(\mathbf{q})+\boldsymbol{\beta} \dot{\mathbf{q}}=\boldsymbol{\tau}+\boldsymbol{\tau}_{c}
$$

In this equation, $\mathbf{q}, \dot{\mathbf{q}} \in \mathbb{R}^{n}$ are the generalized position and velocity vectors, $\mathbf{M}(\mathbf{q}) \in \mathbb{R}^{n \times n}$ is the generalized inertia matrix, $\mathbf{C}(\mathbf{q}, \dot{\mathbf{q}}) \in \mathbb{R}^{n \times n}$ is the Coriolis and centripetal effects matrix, $\mathbf{g}(\mathbf{q}) \in \mathbb{R}^{n}$ is the gravitational torque vector, $\boldsymbol{\beta} \in \mathbb{R}^{n \times n}$ is a viscous friction operator, $\boldsymbol{\tau} \in \mathbb{R}^{n}$ is the vector of control torques, and $\tau_{c} \in \mathbb{R}^{n}$ defines the torque produced by the external forces applied to the robot. Usually, according to the virtual work principle, $\boldsymbol{\tau}_{c}$ is formulated as the additive contribution of the external wrenches ${ }^{0} \mathbf{W}_{j} \in \mathbb{R}^{m}$ applied to the robot body (where $m$ is the task-space dimension), and propagated along its kinematic chain as:

$$
\boldsymbol{\tau}_{c}=\sum_{j=1}^{\gamma} \mathbf{J}_{j}^{\top}(\mathbf{q}){ }^{0} \mathbf{W}_{j}
$$

Each of the $\gamma$ contact points, is associated with a specific Jacobian matrix $\mathbf{J}_{j}(\mathbf{q})=\partial^{0} \mathbf{x}_{a_{j}} / \partial \mathbf{q} \in \mathbb{R}^{m \times n}$, where ${ }^{0} \mathbf{x}_{a_{j}} \in$ $\mathbb{R}^{m}$ represent the actual position of the contact with respect to the base frame of the robot. Hence the Cartesian dynamics ${ }^{0} \ddot{\mathbf{x}}_{a_{j}} \in \mathbb{R}^{m}$ of each contact point can be calculated as:

$$
\begin{aligned}
{ }^{0} \dot{\mathbf{x}}_{a_{j}} & =\mathbf{J}_{j}(\mathbf{q}) \dot{\mathbf{q}} \\
{ }^{0} \ddot{\mathbf{x}}_{a_{j}} & =\dot{\mathbf{J}}_{j}(\mathbf{q}, \dot{\mathbf{q}}) \dot{\mathbf{q}}+\mathbf{J}_{j}(\mathbf{q}) \ddot{\mathbf{q}} \\
& =\dot{\mathbf{J}}_{j}(\mathbf{q}, \dot{\mathbf{q}}) \dot{\mathbf{q}}+\mathbf{J}_{j}(\mathbf{q}) \mathbf{M}(\mathbf{q})^{-\mathbf{1}}\left[\boldsymbol{\tau}+\boldsymbol{\tau}_{c}-\mathbf{h}(\mathbf{q}, \dot{\mathbf{q}})\right]
\end{aligned}
$$

\footnotetext{
${ }^{1}$ The compliance problem is defined with respect to robot base frame where the interaction takes place.
} 

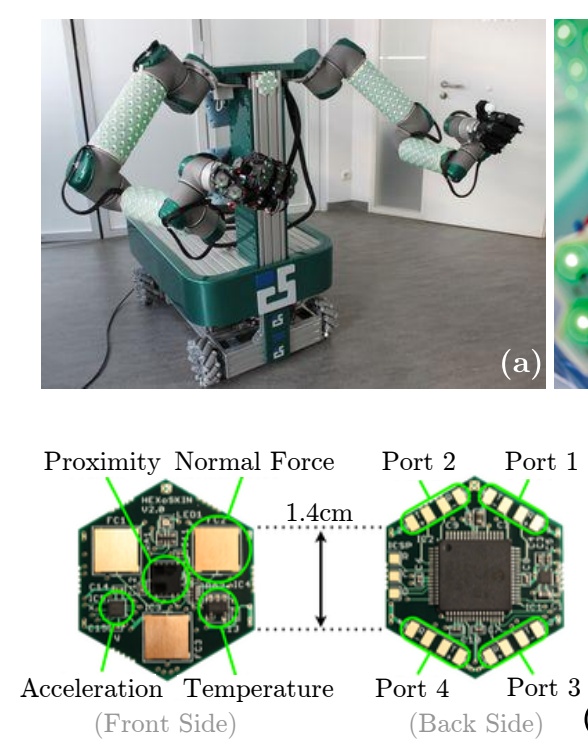
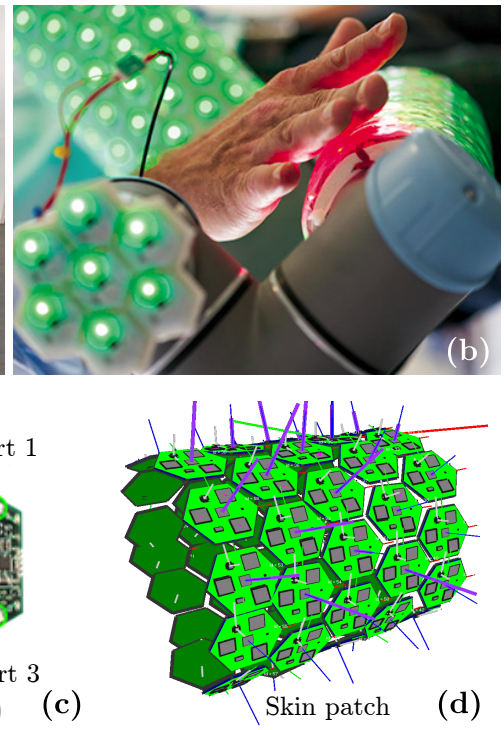

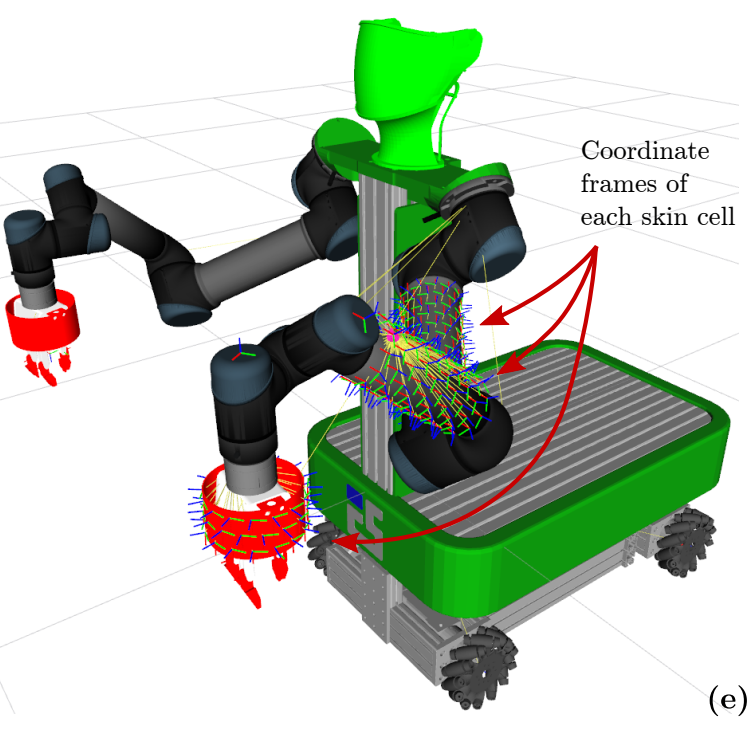

Fig. 1: (a): Our experimental platform, TOMM (Tactile Omnidirectional Mobile Manipulator) [47], is partially covered with a multimodal artificial skin. (b): In this work, the artificial skin is set to react to both proximity and to the normal components of external forces, fusing these informations as a set of cell-specific virtual force vectors ${ }^{0} \mathbf{F}_{\sigma} \in \mathbb{R}^{3}$, given in the base frame of each arm. (c): The artificial skin mounted on TOMM is a redundant network of identical "cells" organized in patches. As presented in [45], each skin cell has a set of three normal force sensors, a temperature sensor, a proximity sensor and a 3D accelerometer. (d): Shape reconstruction of a given skin patch can be achieved after a short self-organization of the cells network followed by a self-calibration step [45]. (e): Once the shape of a skin patch is known, each skin cell can be provided with a specific position vector ${ }^{0} \mathbf{x}_{\sigma} \in \mathbb{R}^{m}$, defined in the base frame of each arm [22]. Notice the corresponding coordinate frames on the left arm of the robot model.

where $\dot{\mathbf{J}}_{j}(\mathbf{q}, \dot{\mathbf{q}}) \in \mathbb{R}^{m \times n}$ is the time derivative of the Jacobian matrix $\mathbf{J}_{j}(\mathbf{q})$, and $\mathbf{h}(\mathbf{q}, \dot{\mathbf{q}})=\mathbf{C}(\mathbf{q}, \dot{\mathbf{q}}) \dot{\mathbf{q}}+\mathbf{g}(\mathbf{q})+\boldsymbol{\beta} \dot{\mathbf{q}} \in \mathbb{R}^{n}$. It is important to note that under these conditions, there is absolutely no guarantee that the robot is safe for the external user since neither the contact impedance nor the robot physical limitations are taken into account in eq. (2). Therefore the control objective is to generate a compliant dynamics for ${ }^{0} \ddot{\mathbf{x}}_{a_{j}}$.

\section{B. Compliance at the Contact Point Level}

In practice, controlling the mechanical impedance of a robot manipulator can be achieved at the joint level [10], at the end-effector level [6] or at the contact point level [50]-[52]. Contact point compliance control (also referred to as contact point impedance control) is of particular relevance in the context of whole-body human-robot interaction since it allows to intuitively and precisely adjust the robot reactive behaviors at any point of its kinematic chain [50]. The corresponding torque-control objective can be formulated as determining the command $\tau^{\star}$ providing a specific contact point $j$, with a desired dynamic behavior ${ }^{0} \ddot{\mathbf{x}}_{d_{j}}$, defined in Cartesian space by the following mass-spring-damper equation of motion:

$$
{ }^{0} \mathbf{W}_{j}=\boldsymbol{\Lambda}_{d_{j}} \Delta^{0} \ddot{\mathbf{x}}_{j}+\mathbf{D}_{d_{j}} \Delta^{0} \dot{\mathbf{x}}_{j}+\mathbf{K}_{d_{j}} \Delta^{0} \mathbf{x}_{j}
$$

In this equation, ${ }^{0} \mathbf{W}_{j} \in \mathbb{R}^{m}$ is the external wrench applied to the contact point $j$ and $\boldsymbol{\Lambda}_{d_{j}}, \mathbf{D}_{d_{j}}, \mathbf{K}_{d_{j}} \in \mathbb{R}_{+}^{m \times m}$ are the corresponding desired inertia, damping and stiffness matrices. These matrices define the contact dynamics. $\Delta^{0} \mathbf{x}_{j}=\left({ }^{0} \mathbf{x}_{d_{j}}-{ }^{0} \mathbf{x}_{r e f_{j}}\right) \in \mathbb{R}^{m}$ and their time derivatives are respectively the positions, velocities, and accelerations errors between the desired mass-spring-damper contact dynamics $\left({ }^{0} \mathbf{x}_{d_{j}},{ }^{0} \dot{\mathbf{x}}_{d_{j}},{ }^{0} \ddot{\mathbf{x}}_{d_{j}}\right)$ - which must be tracked by the robot and its reference $\left({ }^{0} \mathbf{x}_{r e f_{j}},{ }^{0} \dot{\mathbf{x}}_{r e f_{j}},{ }^{0} \ddot{\mathbf{x}}_{r e f_{j}}\right)$, towards which the robot must converge when no force is applied to it. When interaction is limited to a single contact point the resulting compliance control law can be derived as:

$$
\begin{aligned}
\boldsymbol{\tau}^{\star} & =\mathbf{M}(\mathbf{q}) \ddot{\mathbf{q}}_{d}+\mathbf{h}(\mathbf{q}, \dot{\mathbf{q}}) \\
\ddot{\mathbf{q}}_{d} & =\mathbf{J}_{j}^{\#}(\mathbf{q})\left({ }^{0} \ddot{\mathbf{x}}_{d_{j}}-\dot{\mathbf{J}}_{j}(\mathbf{q}, \dot{\mathbf{q}}) \dot{\mathbf{q}}\right)+\mathbf{P}_{j} \ddot{\mathbf{q}}_{N} \\
{ }^{0} \ddot{\mathbf{x}}_{d_{j}} & ={ }^{0} \ddot{\mathbf{x}}_{r e f_{j}}+\boldsymbol{\Lambda}_{d_{j}}^{-1}\left[{ }^{0} \mathbf{W}_{j}-\mathbf{D}_{d_{j}} \Delta^{0} \dot{\mathbf{x}}_{j}-\mathbf{K}_{d_{j}} \Delta^{0} \mathbf{x}_{j}\right]
\end{aligned}
$$

were $\mathbf{J}_{j}^{\#}(\mathbf{q}) \in \mathbb{R}^{n \times m}$ is the generalized inverse of $\mathbf{J}_{j}(\mathbf{q})$, $\mathbf{P}_{j}=\mathbf{I}_{n \times n}-\mathbf{J}_{j}^{\#}(\mathbf{q}) \mathbf{J}_{j}(\mathbf{q}) \in \mathbb{R}^{n \times n}$ is the corresponding nullspace projector, and $\ddot{\mathbf{q}}_{N} \in \mathbb{R}^{n}$ is an arbitrary joint acceleration vector to be projected on the nullspace of $\mathbf{J}_{j}(\mathbf{q})$. This control technique was successfully implemented in [50], using the dynamic residual method [53], [54] for contact force estimation and exploiting visual feedback for contact point localization. Its generalization to scenarios involving simultaneous interaction with $\rho \in[1, n]$ different robot links can be achieved using an augmented Jacobian representation, eventually leading to the following reformulation of eq. (5b):

$$
\ddot{\mathbf{q}}_{d}=\mathbf{J}_{a u g}^{\#}(\mathbf{q})\left({ }^{0} \ddot{\mathbf{x}}_{d_{a u g}}-\dot{\mathbf{J}}_{a u g}(\mathbf{q}, \dot{\mathbf{q}}) \dot{\mathbf{q}}\right)+\mathbf{P}_{a u g} \ddot{\mathbf{q}}_{N}
$$

where $\mathbf{J}_{\text {aug }}=\left[\begin{array}{lll}\mathbf{J}_{1}^{\top} & \cdots & \mathbf{J}_{\rho}^{\top}\end{array}\right]^{\top} \in \mathbb{R}^{\rho m \times n}$ concatenates the $\rho$ Jacobian matrices of the reference interaction points,

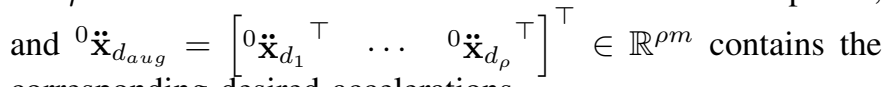
corresponding desired accelerations. 
(a)

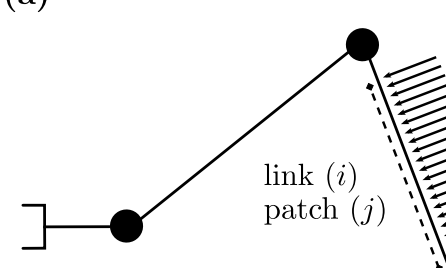

(b)

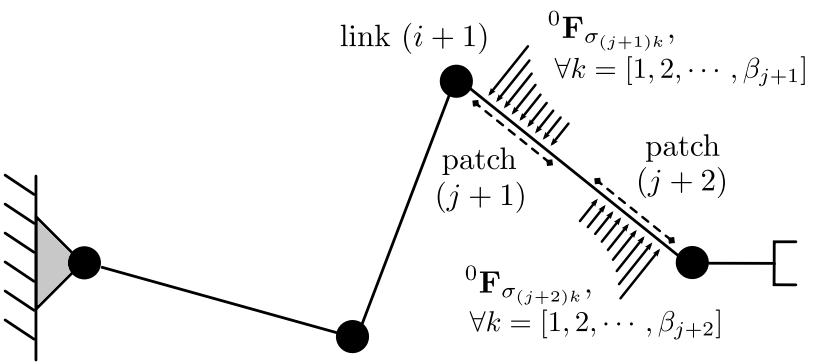

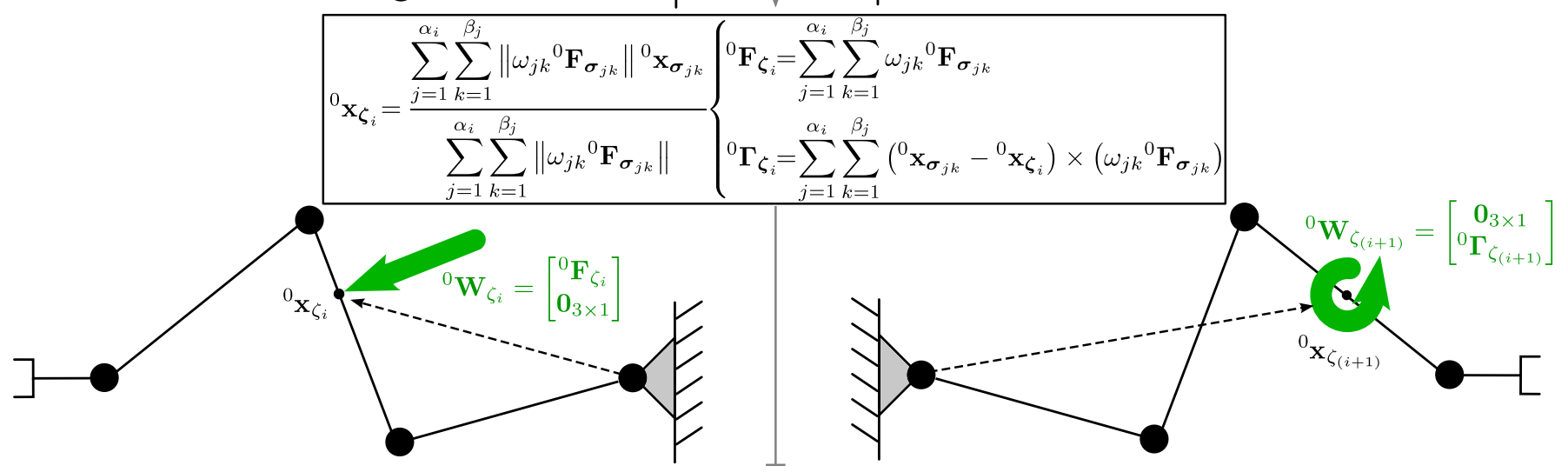

Fig. 2: Illustration of the equivalence between a given normal force distribution - measured by the artificial skin - and a single wrench applied to the force centroid of the considered rigid body. In this example, we consider a simplified model of plane robot with 4 degrees of freedom. (a) depicts the case of a single skin patch $j$, mounted on the robot link $i$, and reading a set of $\beta_{j}$ different cell forces ${ }^{0} \mathbf{F}_{\boldsymbol{\sigma}_{j k}}$. The resulting wrench ${ }^{0} \mathbf{W}_{\boldsymbol{\zeta}_{i}}$ applied to the force centroid ${ }^{0} \mathbf{x}_{\zeta_{i}}$ has no torque components since the applied forces have the same direction. (b) depicts the case of two different skin patches mounted on the same rigid body and submitted to a set of counteracting forces with equivalent magnitude. In this case, the resulting wrench has no translational components.

\section{Whole-Body Multi-Contact Compliance UNDER CONSTRAINTS}

\section{A. Tactile Multiple-Contact Compliance}

When contact with the external environment simultaneously occurs at multiple locations of given robot link, the combined action of the resulting interaction forces must be adequately taken into account to generate suitable reactive motions according to eq. (6). In a contact-point compliance scenario, this requires a precise knowledge of both the forces applied to the robot and their localization on its kinematic chain. Since tactile feedback immediately provides information about the type, location, and intensity of an arbitrary number of physical interactions, it is perfectly fitted for this task. In this paper, we merge the artificial skin data into a single wrench vector, applied to the centroid of the considered link force distribution. The concept of force centroid can be thought of as a threedimensional version of the zero moment point (ZMP) of a walking robot [55], [56], defined as the weighted average location $\mathbf{x}_{z m p}$ of the pressure efforts $\mathbf{p}(\mathbf{x}), \mathbf{x} \in \Omega$ applied to its feet:

$$
\mathbf{x}_{z m p}=\frac{\int_{\Omega} \mathbf{x p}(\mathbf{x}) d \mathbf{x}}{\int_{\Omega} \mathbf{p}(\mathbf{x}) d \mathbf{x}}
$$

In our case, the location ${ }^{0} \mathbf{x}_{\zeta_{i}}$ of the force centroid related with the rigid link $i$, is defined as a cell-wise discretized version of eq. (7). Let us consider the serial manipulator depicted in Fig. 2, whose rigid links $i$ are covered by a number $\alpha_{i}$ of skin patches. Each patch $j=\left[1,2, \cdots \alpha_{i}\right]$ of artificial skin contains a set of $\beta_{j}$ activated skin cells, sending a stream of relevant force ${ }^{0} \mathbf{F}_{\boldsymbol{\sigma}_{j k}} \in \mathbb{R}^{3}$ and position ${ }^{0} \mathbf{x}_{\boldsymbol{\sigma}_{j k}} \in \mathbb{R}^{3}$ data. Then the set of all the ${ }^{0} \mathbf{F}_{\boldsymbol{\sigma}_{j k}}$ applied to the corresponding contact points ${ }^{0} \mathbf{x}_{\boldsymbol{\sigma}_{j k}}$ can be seen as equivalent to a unique wrench ${ }^{0} \mathbf{W}_{\boldsymbol{\zeta}_{i}}=\left[{ }^{0} \mathbf{F}_{\boldsymbol{\zeta}_{i}}^{\top},{ }^{0} \boldsymbol{\Gamma}_{\boldsymbol{\zeta}_{i}}^{\top}\right]^{\top}$ applied to the force centroid ${ }^{0} \mathbf{x}_{\zeta_{i}}$ of the rigid body $i$ where:

$$
\begin{aligned}
&{ }^{0} \mathbf{x}_{\boldsymbol{\zeta}_{i}}= \frac{\sum_{j=1}^{\alpha_{i}} \sum_{k=1}^{\beta_{j}} \omega_{j k}\left\|{ }^{0} \mathbf{F}_{\boldsymbol{\sigma}_{j k}}\right\|{ }^{0} \mathbf{x}_{\boldsymbol{\sigma}_{j k}}}{\sum_{j=1}^{\alpha_{i}} \sum_{k=1}^{\beta_{j}} \omega_{j k}\left\|{ }^{0} \mathbf{F}_{\boldsymbol{\sigma}_{j k}}\right\|} \\
&{ }^{0} \mathbf{F}_{\boldsymbol{\zeta}_{i}}=\sum_{j=1}^{\alpha_{i}} \sum_{k=1}^{\beta_{j}} \omega_{j k}{ }^{0} \mathbf{F}_{\boldsymbol{\sigma}_{j k}} \\
&{ }^{0} \boldsymbol{\Gamma}_{\boldsymbol{\zeta}_{i}}=\sum_{j=1}^{\alpha_{i}} \sum_{k=1}^{\beta_{j}}\left({ }^{0} \mathbf{x}_{\boldsymbol{\sigma}_{j k}}-{ }^{0} \mathbf{x}_{\boldsymbol{\zeta}_{i}}\right) \times\left(\omega_{j k}{ }^{0} \mathbf{F}_{\boldsymbol{\sigma}_{j k}}\right)
\end{aligned}
$$

An important point to be noted in the last equations is the cellwise weighting factor $\omega_{j k} \in \mathbb{R}_{+}^{\star}$, which allows implementing spatial variations in cell sensitivity within the same skin patch. This is an important feature of the artificial skin used in this work, which makes it possible to emulate the changes in 
sensory density observed on the natural skin. In this manner, the behavior of a robot can not only be adjusted limb-wise but also according to the location of interaction as it is, in particular, the case for humans ${ }^{2}$. Using this formulation, the centroid interaction wrenches ${ }^{0} \mathbf{W}_{\boldsymbol{\zeta}_{i}}$ measured by the skin can be directly re-injected into eq. (5c) to generate a set of desired acceleration vectors ${ }^{0} \ddot{\mathbf{x}}_{d_{\zeta_{i}}}$ (one for each link involved in the interaction process). An adequate torque command can then be issued using eq. (6) and eq. (5a). In this case the augmented variables are obtained by concatenating the corresponding centroid quantities, for example $\mathbf{J}_{\text {aug }}=\left[\begin{array}{lll}\mathbf{J}_{\boldsymbol{\zeta}_{1}}^{\top} & \cdots & \mathbf{J}_{\boldsymbol{\zeta}_{\rho}}^{\top}\end{array}\right]^{\top} \in$ $\mathbb{R}^{\rho m \times n}$ where $\mathbf{J}_{\boldsymbol{\zeta}_{i}}$ is the Jacobian matrix associated with the centroid $i$ and $\rho \in[1, n]$ is the total number of centroids. Special attention must here be given to the computation of $\dot{\mathbf{J}}_{\text {aug }}(\mathbf{q}, \dot{\mathbf{q}})$ as the evolution of $\mathbf{J}_{\boldsymbol{\zeta}_{i}}(\mathbf{q})$ does not only depends on the robot motions but also on the changes of centroid position in the considered rigid body. We here approximate $\dot{\mathbf{J}}_{\boldsymbol{\zeta}_{i}}(\mathbf{q}, \dot{\mathbf{q}})$ using a first order Taylor expansion with the control loop time step $\delta t$ :

$$
\begin{aligned}
\dot{\mathbf{J}}_{\boldsymbol{\zeta}_{i}}(\mathbf{q}, \dot{\mathbf{q}}) & \approx \frac{1}{\delta t}\left[\mathbf{J}_{\boldsymbol{\zeta}_{i}}(\mathbf{q}+\delta \mathbf{q})-\mathbf{J}_{\boldsymbol{\zeta}_{i}}(\mathbf{q})\right] \\
& =\frac{1}{\delta t}\left[\mathbf{J}_{\boldsymbol{\zeta}_{i}}(\mathbf{q}+\dot{\mathbf{q}} \cdot \delta t)-\mathbf{J}_{\boldsymbol{\zeta}_{i}}(\mathbf{q})\right]
\end{aligned}
$$

This link-wise formulation of the robot compliance control law is relevant since the computational complexity no longer depends on the number of activated skin cells as it was the case in our previous approach [48]. However, in its current form, it does not account for the physical constraints of the considered robot.

\section{B. Force Propagation for Whole-Body Tactile Compliance}

1) Computation of the limb total wrench residual: When an external wrench ${ }^{0} \mathbf{W}_{\boldsymbol{\zeta}_{i}}$ applied to a given robot limb, cannot produce the Cartesian acceleration ${ }^{0} \ddot{\mathbf{x}}_{d_{\zeta_{i}}}$ required to match the desired contact impedance at the corresponding force centroid $\zeta_{i}$, a local acceleration residual ${ }^{0} \ddot{\mathbf{x}}_{r_{\zeta_{i}}}$ is generated. This residual can be computed as the difference between the desired acceleration ${ }^{0} \ddot{\mathbf{x}}_{d_{\zeta_{i}}}$ of the mass-spring-damper system submitted to the centroid wrench ${ }^{0} \mathbf{W}_{\boldsymbol{\zeta}_{i}}$, and the acceleration ${ }^{0} \ddot{\mathbf{x}}_{a_{\zeta_{i}}}$ the robot limb can locally generate without violating its physical limitations:

$$
\begin{aligned}
& { }^{0} \ddot{\mathbf{x}}_{r_{\zeta_{i}}}={ }^{0} \ddot{\mathbf{x}}_{d_{\zeta_{i}}}-{ }^{0} \ddot{\mathbf{x}}_{a_{\zeta_{i}}} \\
& \quad={ }^{0} \ddot{\mathbf{x}}_{d_{\zeta_{i}}}-\dot{\mathbf{J}}_{\boldsymbol{\zeta}_{i}}(\mathbf{q}, \dot{\mathbf{q}}) \dot{\mathbf{q}}-\mathbf{J}_{\boldsymbol{\zeta}_{i}}(\mathbf{q}) \mathbf{M}^{-\mathbf{1}}(\mathbf{q})\left[\boldsymbol{\tau}^{\star}-\mathbf{h}(\mathbf{q}, \dot{\mathbf{q}})\right]
\end{aligned}
$$

One of the main challenges is here to correctly propagate these components onto the parent limbs in order to generate suitable reactive behaviors. One way to proceed would be to consider the residuals as local measures of the acceleration of a rigid body. Following the approach developed in [57], it would then be possible to express the total child limb residual acceleration with respect to a parent frame, as an optimization problem. However under these conditions, a proper solution could only be obtained provided that set of - at least - four distinct acceleration residuals is available. If this condition

\footnotetext{
${ }^{2}$ Such a position-dependent weighting factor cannot be obtained using joint force-torque sensors. In this work, we set these weighting factors to 1 .
}

is not verified, the problem may have an infinite number of solutions. Furthermore, the direct propagation of acceleration residuals on a parent limb may also lead to the generation of jerky or counter intuitive behaviors, especially in case of significant mismatch between parent and child dynamics. To solve these issues, we decided to formulate the residual propagation problem at the force level and thus to convert the different ${ }^{0} \ddot{\mathbf{x}}_{r_{\zeta_{i}}}$ into a set of wrench residuals ${ }^{0} \mathbf{W}_{r_{\zeta_{i}}}$ using the desired dynamics $\boldsymbol{\Lambda}_{d_{\zeta_{i}}}$ of the considered link centroid $\boldsymbol{\zeta}_{i}$ :

$$
{ }^{0} \mathbf{W}_{r_{\zeta_{i}}}=\left[{ }^{0} \mathbf{F}_{r_{\zeta_{i}}}{ }^{0} \boldsymbol{\Gamma}_{r_{\zeta_{i}}}\right]=\boldsymbol{\Lambda}_{d_{\zeta_{i}}}{ }^{0} \ddot{\mathbf{x}}_{r_{\zeta_{i}}}
$$

These residuals contain the components of the centroid wrenches that were not adequately compensated on the considered limb. They have to be merged into a single limb wrench residual ${ }^{0} \mathbf{W}_{r}$ applied to the parent-child junction:

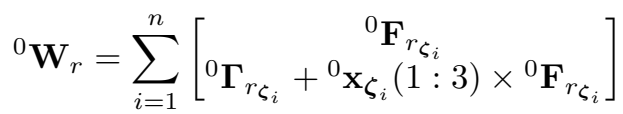

2) Propagation of the limb total wrench residual: The next step is to propagate ${ }^{0} \mathbf{W}_{r}$ onto the considered parent limb and therefore to express it on the corresponding basis. This can be achieved using the adjoint transform

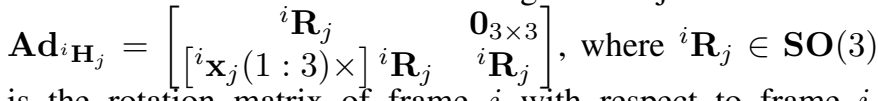
is the rotation matrix of frame $j$ with respect to frame $i$, and $\left[{ }^{i} \mathbf{x}_{j}(1: 3) \times\right] \in \mathbb{R}^{3 \times 3}$ is the skew operator associated with the first three components of the position vector ${ }^{i} \mathbf{x}_{j}$ : $\forall \mathbf{u} \in \mathbb{R}^{3}:\left[{ }^{i} \mathbf{x}_{j}(1: 3) \times\right] \mathbf{u}={ }^{i} \mathbf{x}_{j}(1: 3) \times \mathbf{u}$. Since a given parent limb may have several child limbs sharing the same root frame (e.g. arms mounted on a rigid torso), the expression of the residual wrench applied to this frame must therefore be the sum of these limbs contributions:

$$
{ }^{p} \mathbf{W}_{r}=\sum_{c=1}^{\kappa} \operatorname{Ad}_{p} \mathbf{H}_{0_{c}}{ }^{0_{c}} \mathbf{W}_{r_{c}}
$$

where $\kappa$ is the number of child limbs attached to the same parent link (c.f. Fig.3.c). There may of course be several root links within a considered parent limb. The residual wrench ${ }^{p} \mathbf{W}_{r}$ will therefore have to be calculated for each of these links before being classically re-injected into the parent limb controller as:

$$
\begin{aligned}
& \boldsymbol{\tau}^{\star}=\mathbf{M}_{p}(\mathbf{q}) \ddot{\mathbf{q}}_{d}+\mathbf{h}_{p}(\mathbf{q}, \dot{\mathbf{q}}) \\
& \ddot{\mathbf{q}}_{d}=\mathbf{J}_{a u g}^{\#}(\mathbf{q})\left({ }^{p} \ddot{\mathbf{x}}_{d_{a u g}}-\dot{\mathbf{J}}_{a u g}(\mathbf{q}, \dot{\mathbf{q}}) \dot{\mathbf{q}}\right) \\
& +\mathbf{P}_{\text {aug }} \mathbf{J}_{r_{a u g}}^{\#}(\mathbf{q})(\underbrace{\left[\begin{array}{cc}
\boldsymbol{\Lambda}_{d_{1}}^{-1} & p \\
\vdots \\
\boldsymbol{\Lambda}_{d_{\chi}}^{-1} & p \\
\mathbf{W}_{r_{1}} \\
\mathbf{r}_{\chi}
\end{array}\right]}_{\text {child limbs residual }}-\dot{\mathbf{J}}_{r_{\text {aug }}}(\mathbf{q}, \dot{\mathbf{q}}) \dot{\mathbf{q}})
\end{aligned}
$$

where as previously $\mathbf{J}_{a u g}=\left[\begin{array}{lll}\mathbf{J}_{\boldsymbol{\zeta}_{1}}^{\top} & \cdots & \mathbf{J}_{\boldsymbol{\zeta}_{\rho}}^{\top}\end{array}\right]^{\top} \in \mathbb{R}^{\rho m \times n}$ concatenates the $\rho \in[1, n]$ Jacobian matrices of the reference interaction points (this time in the parent limb) and ${ }^{p} \ddot{\mathbf{x}}_{d_{a u g}}=$ $\left[\begin{array}{lll}{ }^{p} \ddot{\mathbf{x}}_{d_{1}}{ }^{\top} & \ldots & p_{\ddot{\mathbf{x}}_{d_{\rho}}}{ }^{\top}\end{array}\right]^{\top} \in \mathbb{R}^{\rho m}$ contains the corresponding desired accelerations of the parent centroids. 
(a)

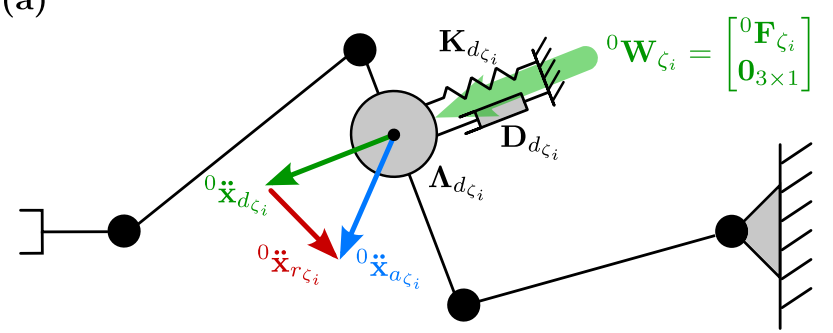

(b)
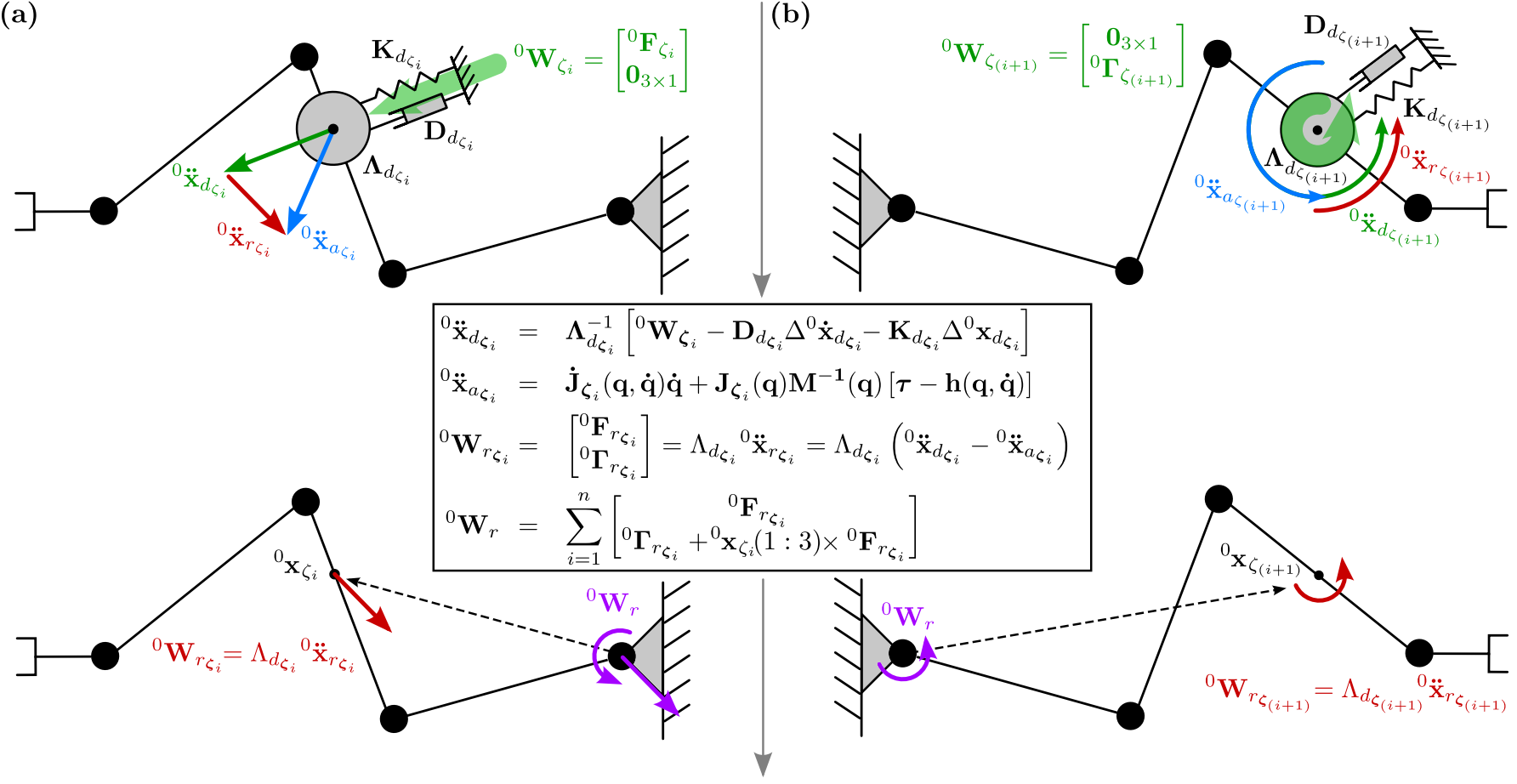

(c)

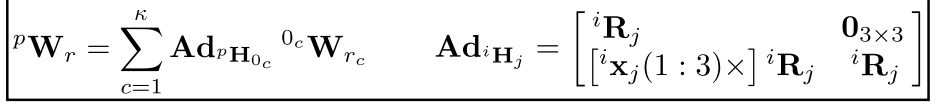

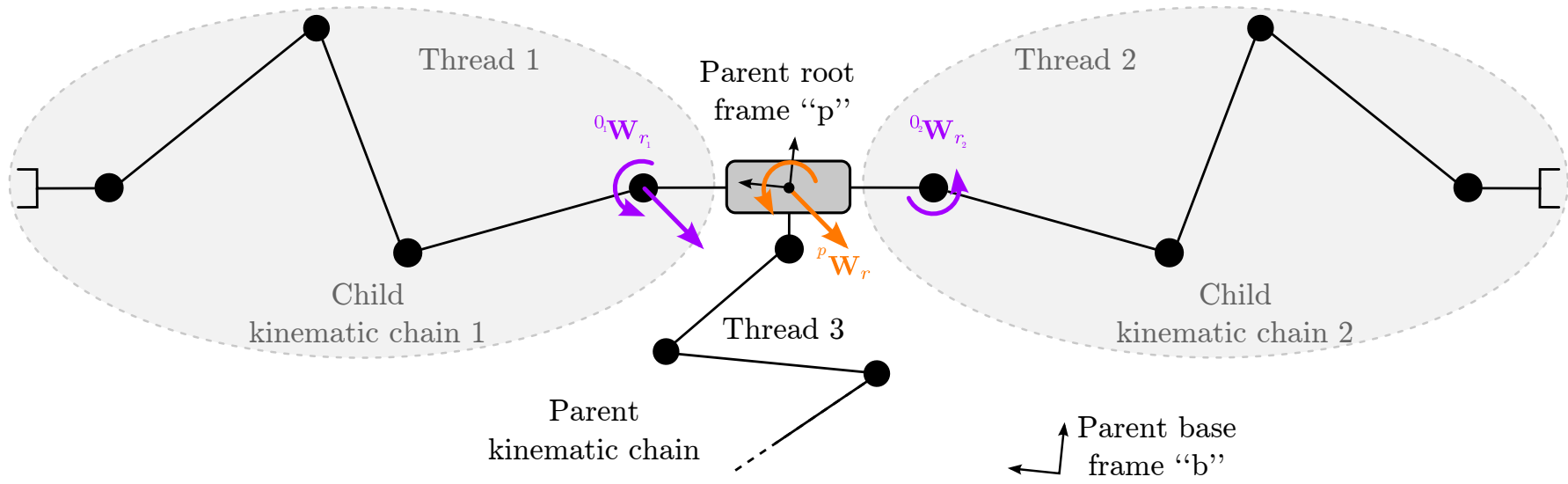

Fig. 3: Illustration of tactile-based multicontact compliance with generalized force propagation. (a-b): Once the wrench ${ }^{0} \mathbf{W}_{\boldsymbol{\zeta}_{i}}$ has been extracted from the skin data on a given link of the robot, it is applied to a virtual mass-spring-damper system, thereby generating a desired local acceleration ${ }^{0} \ddot{\mathbf{x}}_{d_{\zeta_{i}}}$ which must be tracked by the robot as accurately as possible. The errors ${ }^{0} \ddot{\mathbf{x}}_{r_{\zeta_{i}}}$ between the desired and the actual centroid accelerations are a direct consequence of the robot physical constraints. (c): These errors are then locally transformed into a set of centroid wrench residuals ${ }^{0} \mathbf{W}_{r_{\zeta_{i}}}$, which are in turn summed up link-wise to obtain the limb residual wrench ${ }^{0_{i}} \mathbf{W}_{r_{i}}$. The resulting wrench residual ${ }^{p} \mathbf{W}_{r_{i}}$ of each arm can eventually be propagated on the parent kinematic chain (in our case the robot base), in the same manner as an external wrench applied to "p".

In this case, $n$ denotes the dimension of the parent joint space. The residual is propagated in the nullspace of the parent limb interaction task, using the corresponding projector $\mathbf{P}_{\text {aug }}=$ $\mathbf{I}_{n \times n}-\mathbf{J}_{a u g}^{\#}(\mathbf{q}) \mathbf{J}_{\text {aug }}(\mathbf{q}) \in \mathbb{R}^{n \times n}$. The augmented Jacobian $\mathbf{J}_{r_{a u g}}(\mathbf{q})=\left[\begin{array}{lll}\mathbf{J}_{r_{1}}^{\top} & \cdots & \mathbf{J}_{r_{\chi}}^{\top}\end{array}\right]^{\top} \in \mathbb{R}^{\chi m \times n}$ concatenates the $\chi$ Jacobian matrices of the residuals, $\chi$ being the number of links - within the parent limb - that have at least one child limb with a non zero residual. In this case, $\boldsymbol{\Lambda}_{d_{i}} \in \mathbb{R}^{m \times m}$ denotes the desired inertia matrix of parent link $i$.

\section{Tactile Multi-Contact Compliance Under Constraints}

Reformulating the issue of tactile multi-contact compliance as a quadratic optimization problem has many advantages. In particular, it allows to take into account the robot physical constraints directly within the control law, provided that they can be formulated as a set of equality or inequality constraints. In practice, the compliance control law (5) can be thought of as finding the optimal control torque $\tau^{\star}$ minimizing the acceleration residual ${ }^{0} \ddot{\mathbf{x}}_{r_{\zeta_{i}}}^{\top}$, computed in eq. (10). From there 
it is straightforward to formulate the corresponding objective function as the regularized weighted squared $L 2$-norm of the acceleration residual ${ }^{0} \ddot{\mathbf{x}}_{r_{\boldsymbol{\zeta}_{i}}^{\top}}^{\top}$ :

$$
\boldsymbol{\tau}^{\star}=\underset{\boldsymbol{\tau}}{\arg \min } \sum_{i=1}^{n}{ }^{0} \ddot{\mathbf{x}}_{r_{\boldsymbol{\zeta}_{i}}^{\top}}^{\top} \boldsymbol{\Psi}_{i}{ }^{0} \ddot{\mathbf{x}}_{r_{\boldsymbol{\zeta}_{i}}}+\varepsilon \boldsymbol{\tau}^{\top} \boldsymbol{\tau}
$$

In this equation, the variable $\varepsilon \in \mathbb{R}$ is a regularization term, allowing to avoid numerical singularities. This value is automatically set by the optimizer [58]. The weighting matrix $\boldsymbol{\Psi}_{i}=\operatorname{diag}\left(\psi_{i j}\right) \in \mathbb{R}_{+}^{m}$ plays an important role, by either enhancing or penalizing the influence of linear (respectively angular) components of ${ }^{0} \ddot{\mathbf{x}}_{r_{\zeta_{i}}}^{\top}$ within the global optimization process $^{3}$. This is essential since the minimization of the linear acceleration residual is sometimes achieved at the expense of the angular acceleration residual. In this case, the total wrench residual ${ }^{0} \mathbf{W}_{r}$ of eq. (12) has to be reformulated as:

$$
{ }^{0} \mathbf{W}_{r}=\iota \sum_{i=1}^{n}\left[\begin{array}{c}
\overline{\boldsymbol{\Psi}}_{i}{ }^{0} \mathbf{F}_{r_{\zeta_{i}}} \\
\underline{\boldsymbol{\Psi}}_{i}{ }^{0} \boldsymbol{\Gamma}_{r_{\zeta_{i}}}+{ }^{0} \mathbf{x}_{\boldsymbol{\zeta}_{i}}(1: 3) \times \overline{\boldsymbol{\Psi}}_{i}{ }^{0} \mathbf{F}_{r_{\zeta_{i}}}
\end{array}\right]
$$

where $\overline{\boldsymbol{\Psi}}_{i}=\boldsymbol{\Psi}_{i}(1: 3,1: 3), \underline{\boldsymbol{\Psi}}_{i}=\boldsymbol{\Psi}_{i}(4: 6,4: 6)$, and $\iota \in \mathbb{R}_{+}$is a tuning parameter.

\section{Robot Physical Limitations}

As every mechanical system, robots are submitted to a set of kinematic or dynamic constraints, in particular, due to their geometry or actuation configuration. When a robot reaches one of its mechanical limits, the torque applied to the considered joints is automatically set to zero by internal safety checks, resulting in a temporary loss of one or several degrees of freedom. In practice, this is a risk to the external user since the controller is not aware that the robot can no longer move in a given direction of space. Nevertheless, maintaining compliance in this context is still possible, provided that the robot's constraints are properly taken into account in the main control loop. In this way, the forces applied in the vicinity of a mechanical limit can be identified and directly projected onto a parent limb (e.g. the mobile base), thereby generating suitable compliant reactive motions. Since most robots kinematic and dynamic constraints can be formulated as a set of inequalities, they can, therefore, be naturally taken into account in the previous QP formulation. As proposed in [59], we here consider a set of joint space constraints, namely torque, joint, and velocity limits:

$$
\begin{aligned}
-\boldsymbol{\tau}_{u} & \leq \boldsymbol{\tau}^{\star} \leq \boldsymbol{\tau}_{u} \\
\lambda\left(\mathbf{q}_{l}-\mathbf{q}\right) & \leq \boldsymbol{\tau}^{\star} \leq \lambda\left(\mathbf{q}_{u}-\mathbf{q}\right) \\
\mu\left(\dot{\mathbf{q}}_{l}-\dot{\mathbf{q}}\right) & \leq \boldsymbol{\tau}^{\star} \leq \mu\left(\dot{\mathbf{q}}_{u}-\dot{\mathbf{q}}\right)
\end{aligned}
$$

where $\lambda, \mu \in \mathbb{R}$ are convergence rates and where $\mathbf{q}_{l}, \mathbf{q}_{u} \dot{\mathbf{q}}_{l}$ and $\dot{\mathbf{q}}_{u} \in \mathbb{R}^{n}$ are lower and upper joint position and velocity limits vectors for the robot's upper kinematic chains. Both convergence rates $\lambda$ and $\mu$ were chosen heuristically. The

${ }^{3}$ This is equivalent to the use of a weighted pseudo-inverse in eq. (6).
QP for the constrained multi-contact compliance problem can eventually be formulated as:

$$
\begin{array}{lrl}
\boldsymbol{\tau}^{\star}=\underset{\boldsymbol{\tau}}{\arg \min } & \sum_{i=1}^{n}{ }^{0} \ddot{\mathbf{x}}_{r_{\boldsymbol{\zeta}_{i}}}^{\top} \boldsymbol{\Psi}_{i}{ }^{0} \ddot{\mathbf{x}}_{r_{\zeta_{i}}}+\varepsilon \boldsymbol{\tau}^{\top} \boldsymbol{\tau} \\
& -\boldsymbol{\tau}_{u} \leq \boldsymbol{\tau}^{\star} \leq \boldsymbol{\tau}_{u} \\
& \lambda\left(\mathbf{q}_{l}-\mathbf{q}\right) \leq \boldsymbol{\tau}^{\star} \leq \lambda\left(\mathbf{q}_{u}-\mathbf{q}\right) \\
& \mu\left(\dot{\mathbf{q}}{ }_{l}-\dot{\mathbf{q}}\right) \leq \boldsymbol{\tau}^{\star} \leq \mu\left(\dot{\mathbf{q}}_{u}-\dot{\mathbf{q}}\right)
\end{array}
$$

When one of the robot limbs approaches a mechanical limit, the torque generated by the external forces onto the considered joints is automatically bounded. The QP-solver then iteratively searches for the control torque that best matches the objective function while complying with these new constraints. In our approach, if the solver fails to find such a solution, the generalized forces applied to the considered limb will be directly propagated to a parent kinematic chain by setting $\boldsymbol{\tau}^{\star}=\mathbf{0}_{n \times 1}$ in eq. 10. It is important to notice that when interaction occurs between the robot and its environment, the rigid links that are involved in the interaction process have strict priority over those which are not in contact with the environment. In practice, this is achieved by setting the acceleration residual ${ }^{0} \ddot{\mathbf{x}}_{r_{\zeta_{i}}}^{\top}$ of the considered links to zero in eq. (15) and (18).

\section{EXPERIMENTAL EVALUATION}

\section{A. Description of the Hardware and Control Interface}

1) Description of the robot hardware: The experimental setup is presented in Fig. 1.a. Our robot platform TOMM (Tactile Omni-directional Mobile Manipulator) [47] is a dual arm mobile manipulator, composed of two UR5 industrial robots with 6 degrees of freedom each, covered with multimodal artificial skin and mounted on a holonomic mobile base (Fig. 1.a). TOMM's central unit is composed of two intel Core i7-4702MQ computers with $16 \mathrm{~Gb}$ of DDR3 RAM each. One of these CPU is fully dedicated to the control tasks. Both computers operate under Linux Ubuntu 14.04.5 LTS. The complete control stack, including linear algebra and optimization libraries is coded in C++. Real-time capability is here obtained using the Linux PREEMPT_RT kernel patch.

2) Assumptions: Similar to the "floating base" framework of humanoid robots, and disregarding the issues of tilting and instability, we model the omnidirectional mobile base coupled to its low level velocity controller, as a 3-DoF robot made of two orthogonal prismatic joints moving in the horizontal plane and one revolute joint, rotating along the vertical axis. We moreover consider the mobile base and the upper limbs as separated systems. The arm-arm and arm-base dynamic couplings are especially not taken into account. Although this assumption is constraining, it nevertheless remains valid in our case since the torso is not articulated, the mass of the mobile base is one order of magnitude higher than that of the arms, that the omnidirectional wheels are not mounted on shock absorbers and that they also generate significant friction on the ground. Moreover, since the arms of the robot are industrial grade manipulators, we assume that the disturbances generated by the mobile base accelerations are negligible. The fully coupled system is to be considered in future works. 
3) Description of the control interface: TOMM's arms are velocity-controlled (similarly to most industrial robots). However, model-based force/torque control strategies can still be implemented through a set of admittance controllers, converting the generated torque commands into suitable velocity commands as demonstrated in [22]. The control loop frequency is here fixed to $125 \mathrm{~Hz}$ by the real-time interface used to communicate with each UR5 robot arms ${ }^{4}$. A specific admittance controller - similar to [4] - was also developed for our mobile base, in order to make it force/torque-controllable, thereby allowing force propagation from the arms to the mobile base. The virtual dynamics of the mobile base is described by the following three-dimensional equation of motion:

$$
\mathbf{M}_{b} \dot{\mathbf{v}}_{b}+\mathbf{D}_{b} \mathbf{v}_{b}=\mathbf{S}_{b}^{p} \mathbf{W}_{r}
$$

where $\dot{\mathbf{v}}_{b}=\mathbf{S}_{b} \ddot{\mathbf{x}} \in \mathbb{R}^{3}$ concatenates the first two linear components and the last angular component of a Cartesian acceleration and $\mathbf{S}_{b}=\left[\begin{array}{llll}1 & 0 & & 0 \\ 0 & 1 & \mathbf{0}_{3 \times 3} & 0 \\ 0 & 0 & & 1\end{array}\right]$ is the corresponding selection matrix. The parameters $\mathbf{M}_{b}=\operatorname{diag}(m, m, I) \in$ $\mathbb{R}^{3 \times 3}$ and $\mathbf{D}_{b}=\operatorname{diag}\left(d_{1}, d_{2}, d_{3}\right) \in \mathbb{R}^{3 \times 3}$ represent the virtual inertia and damping of the admittance controller, where $m \in \mathbb{R}_{+}$is the mobile base virtual mass, $I \in \mathbb{R}_{+}$is its virtual moment of inertia and $d_{1}, d_{2}, d_{3} \in \mathbb{R}_{+}$are its viscous friction coefficients. During the experiments, the virtual damping values were set to $d_{1}=d_{2}=40$ N.s.m $m^{-1}$ and $d_{3}=40$ N.m.s.rad ${ }^{-1}$. These values were chosen heuristically. When the wrench ${ }^{p} \mathbf{W}_{r}$ is applied to the mobile base, it produces a reference Cartesian acceleration $\dot{\mathbf{v}}_{b} \in \mathbb{R}^{3}$. This acceleration is then integrated and sent as a bounded velocity reference to the mobile-base velocity controller each $8 \mathrm{~ms}^{5}$.

4) Description of the artificial skin: The artificial skin used in this paper consists of identical, hexagonally shaped skin cells, connected to each other with flexible PCBs and assembled into patches of different sizes (see Fig. 1.b,c,d). Each skin cell is capable of simultaneously measuring four different tactile modalities, namely pressure (capacitive force sensors [60]), pre-touch (infrared proximity sensor), temperature (temperature sensor) and vibrations (3D accelerometer). The data generated by each skin patch is transmitted via high-speed serial communication to a FPGA module - the Tactile Section Unit (TSU) - before begin broadcasted to the host computer at a maximum frequency of $250 \mathrm{~Hz}^{6}$ via Gigabit Ethernet. In this manner, a temporal resolution of $4 \mathrm{~ms}$ can be achieved. This is similar to the $5 \mathrm{~ms}$ temporal resolution of Human skin. On TOMM, a set of 4 distinct TSU is broadcasting the data from 14 skin patches, for a total of 644 skin cells covering the two arms. Depending on how

\footnotetext{
${ }^{4}$ Note that the UR5 arm torque resolver described in [22] runs on its own real-time thread at $500 \mathrm{~Hz}$.

${ }^{5}$ The internal velocity control loop of the mobile base operates at $1 \mathrm{kHz}$ on its own real-time thread.

${ }^{6}$ The data broadcasted by the skin are stored in arrays of fixed-size data containers, accessible by the main control loop. Each container stores the multi-modal sensory information of one skin cell. These containers are continuously updated by the arrival of new information. When the robot controller requests data, it receives the most recent buffer value. In this manner the artificial skin and the robot controller can operate at different frequencies.
}

"far" a skin cell is from the TSU - i.e. how many skin cells have to be crossed before reaching the TSU - it takes about $1 \mathrm{~ms}$ to propagate the information to the main CPU. A new command is then issued by the controller every $8 \mathrm{~ms}$ using the last informations provided by the skin (note that the control frequency is here determined by the real-time interface used to communicate with each UR5 robot arm). It is interesting to compare this to human tactile reflexes, such as the patellar reflex, which is issued in about $29.6 \pm 6.0 \mathrm{~ms}$ [61].

5) Details of the $Q P$ formulation: We use the qpOASES $\mathrm{C}++$ library [62] as QP solver since it provides useful regularization options as well as suitable timing tools, allowing implementation in real-time control loops. On a more formal point of view, qpOASES requires the problem to be formulated using box constraints, which is very well suited to our implementation:

$$
\begin{array}{cl}
\min _{\mathbf{z}} & \frac{1}{2} \mathbf{z}^{\top} \mathbf{H z}+\mathbf{z}^{\top} \mathbf{b} \\
\text { s.t. } & \mathbf{l b} \leq \mathbf{z} \leq \mathbf{u b}
\end{array}
$$

In this expression, the matrix $\mathbf{H} \in \mathbb{R}^{\vartheta \times \vartheta}$ denotes the Hessian, and the vectors $\mathbf{b}, \mathbf{z}, \mathbf{l b}, \mathbf{u b} \in \mathbb{R}^{\vartheta}$ respectively denotes the gradient, the optimization variable, and its corresponding lower and upper bounds. Here $\vartheta \in[0,6]$ is the dimension of the problem. Note that in this case, equality constraints can still be handled by simply putting the same expression on both sides of the inequality in eq. (20b). In our case ${ }^{7}$ the QP (18) can be formulated as:

$$
\begin{aligned}
\mathbf{z} & =\mathbf{S} \boldsymbol{\tau} \\
\mathbf{H} & =\mathbf{S}\left[\sum_{i=1}^{n}\left(\mathbf{J}_{\boldsymbol{\zeta}_{i}} \mathbf{M}^{-1}\right)^{\top} \boldsymbol{\Psi}_{i} \mathbf{J}_{\boldsymbol{\zeta}_{i}} \mathbf{M}^{-1}\right] \mathbf{S}^{\top} \\
\mathbf{g} & =-\mathbf{S} \sum_{i=1}^{n}\left(\mathbf{J}_{\boldsymbol{\zeta}_{i}} \mathbf{M}^{-1}\right)^{\top} \mathbf{\Psi}_{i}\left[{ }^{0} \ddot{\mathbf{x}}_{d_{\zeta_{i}}}-\dot{\mathbf{J}}_{\boldsymbol{\zeta}_{i}} \dot{\mathbf{q}}\right. \\
& \left.+\mathbf{J}_{\boldsymbol{\zeta}_{i}} \mathbf{M}^{-1} \mathbf{h}\right] \\
\mathbf{u b} & =\mathbf{S} \min \left(\boldsymbol{\tau}_{u}, \lambda\left(\mathbf{q}_{u}-\mathbf{q}\right), \mu\left(\dot{\mathbf{q}}_{u}-\dot{\mathbf{q}}\right)\right) \\
\mathbf{l b} & =\mathbf{S} \max \left(-\boldsymbol{\tau}_{u}, \lambda\left(\mathbf{q}_{l}-\mathbf{q}\right), \mu\left(\dot{\mathbf{q}}_{l}-\dot{\mathbf{q}}\right)\right)
\end{aligned}
$$

where the min and max operators are defined element-wise and where $\mathbf{S}=\left[\begin{array}{ll}\mathbf{I}_{\vartheta \times \vartheta} & \mathbf{0}_{\vartheta \times(n-\vartheta)}\end{array}\right] \in \mathbb{R}^{\vartheta \times n}$ is a selection matrix allowing to discard the influence of unused joints within the QP. For a given kinematic chain, the dimension $\vartheta$ of the QP corresponds to the index of the highest-order joint involved in the interaction process. For instance, a singlecontact interaction with the fourth rigid link of the robot (moving with the third joint), will result in a three-dimensional problem.

\section{B. Description of the Experiments}

1) Summary: A set of five different experiments was conducted in order to demonstrate the main features of the proposed approach. The first experiment aims at illustrating the ability of our control algorithm to comply with a set of joint position constraints. The second experiment aims

\footnotetext{
${ }^{7}$ For the sake of compactness, the $\mathbf{q}, \dot{\mathbf{q}}$ dependencies will be removed in the following equations.
} 
at highlighting the possibility to generate compliant reactive behaviors with an adjustable dynamics, in reaction to multi-contact physical interactions under torque constraints ${ }^{8}$. The third and fourth experiments study the behavior of the proposed control algorithm in the specific cases of fully propagated and nullspace interactions respectively. Finally, the fifth experiment compares the computational efficiency of the proposed method with that of our previous approach in [48].

2) Experiment protocols: The experiments follow the same protocol: forces are applied to different locations of TOMM's arms. To have an effect on the robot, it is required that these forces are applied to a surface which is covered by the artificial skin. In the current configuration, any force applied on an uncovered surface of the robot will not be detected. These forces are then processed according to eq. (8) in order to compute the position ${ }^{0} \mathbf{x}_{\boldsymbol{\zeta}_{i}}$ of the link forcecentroid and the associated interaction wrench ${ }^{0} \mathbf{W}_{\zeta_{i}}$. The next step is to generate a command $\tau^{\star}$ that will satisfy as much as possible the desired reactive behaviors - defined by the mass-spring-damping parameters assigned to each centroid while at the same time complying with the robot physical constraints. This is achieved by solving the QP of eq. (18). The Cartesian acceleration residuals are then computed for each arm according to eq. 10. Once transformed into wrench residuals by injecting them into eq. 11, they are propagated onto the mobile base using eq. (12), (13) and eq. (19). It is important to note that single and dual arm interactions are treated exactly in the same manner since each arm has its own controller instance, running in a specific thread, as exposed in Fig.3.c. In the case of dual arm interaction, the wrench residuals computed on the base frame of each arm, are simply summed up on the parent root frame (denoted as "p" on Fig.3.c) according to eq. (13). The resulting wrench is then propagated to the mobile base.

\section{Results and Discussion}

1) Experiment 1. Robot joint limit avoidance: The objective of this first experiment is to demonstrate that the the proposed QP formulation of the compliance control law allows a convenient handling of the robot physical constraints and in particular of joint limits. The experiment itself consists in interacting with the robot in different contact points. Moving the right arm of TOMM to its home position $(\mathbf{q}=$ $[0, \pi / 2,0, \pi / 2,0,0]^{\top}$ ), we define the following constrains:

- Torque constraints: $-\boldsymbol{\tau}_{u} \leq \boldsymbol{\tau}^{\star} \leq \boldsymbol{\tau}_{u}$ where $\boldsymbol{\tau}_{u}=$ $[5,5,5,5,5,5]^{\top}[\mathrm{N} . \mathrm{m}]$

- Joint limits: $\lambda\left(\mathbf{q}_{l}-\mathbf{q}\right) \leq \boldsymbol{\tau}^{\star} \leq \lambda\left(\mathbf{q}_{u}-\mathbf{q}\right)$ where the convergence rates are set to $\lambda=\mu=7$ and where $\mathbf{q}_{l}=\left[-0.2, \frac{\pi}{2}-0.2,-0.2, \frac{\pi}{2}-0.2,-0.2,-0.2\right]^{\top}[\mathrm{rad}]$ and $\mathbf{q}_{u}=\left[0.2, \frac{\pi}{2}+0.2,0.2, \frac{\pi}{2}+0.2,0.2,0.2\right]^{\top}[\mathrm{rad}]$.

In this experiment, the desired centroid dynamic parameters are as follow: $\boldsymbol{\Lambda}_{d_{\zeta_{3}}}=2.5 \cdot \mathbf{I}_{6 \times 6}, \mathbf{D}_{d_{\zeta_{3}}}=3 \cdot \mathbf{I}_{6 \times 6}$ and $\mathbf{K}_{d_{\zeta_{3}}}=$

\footnotetext{
${ }^{8}$ It is important to note that although this experiment specifically considers the case of high stiffness reactive dynamics, a wide variety of different dynamic responses can also be generated. Some of these behaviors can be observed within the experiments 1 (zero stiffness) and 3-4 (medium stiffness) for which different values of the contact mass-spring-damper constants were investigated as well as different mobile base dynamic parameters.
}
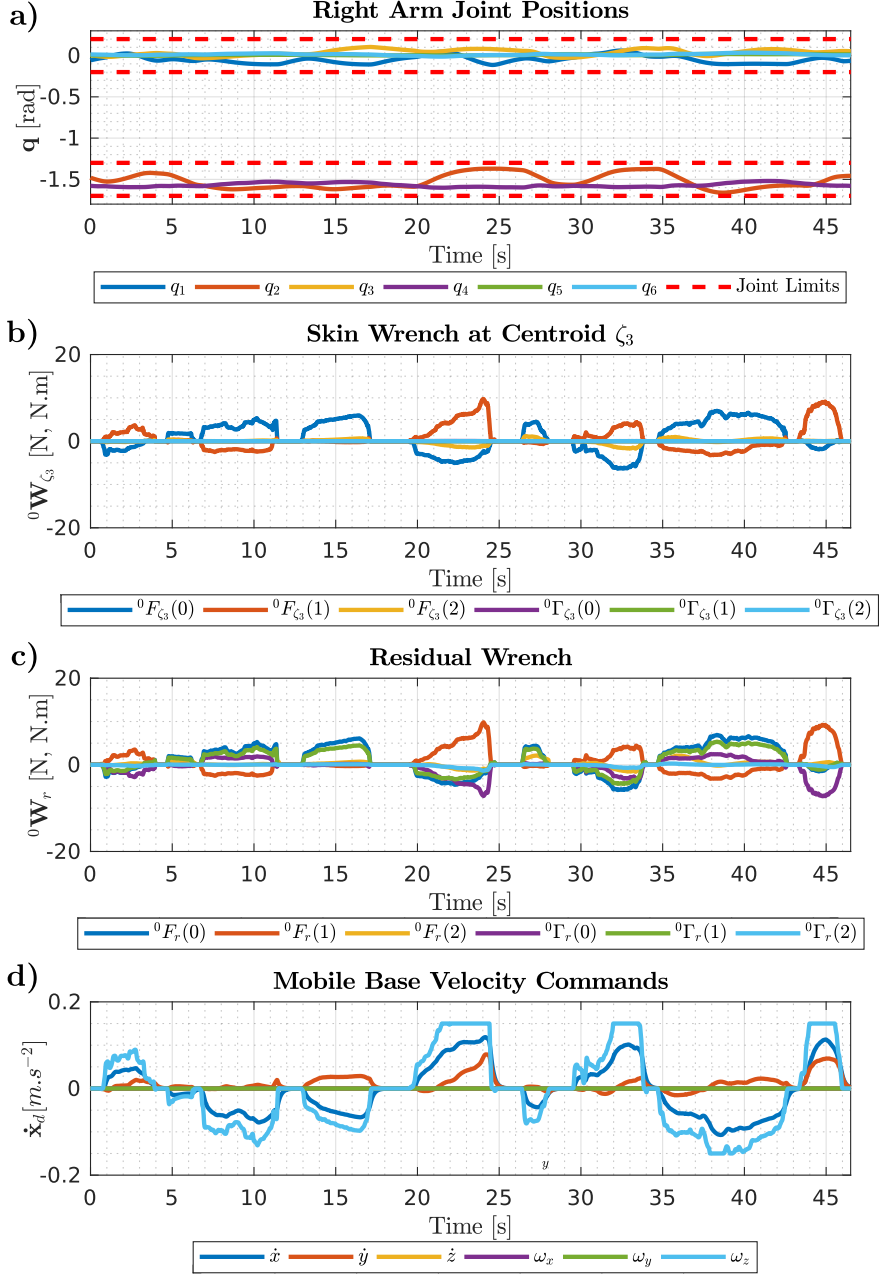

Fig. 4: Robot joint limit avoidance

$\mathbf{0}_{6 \times 6}$. The mobile base virtual mass is set to $m=10 \mathrm{~kg}$ and its virtual inertia to $I=1.0 \mathrm{~kg} . \mathrm{m}^{2}$. The results, shown in Fig. 4 depict that the previously defined constraints are respected: the considered interaction wrench ${ }^{0} \mathbf{W}_{\zeta_{3}}$ located in a centroid $\zeta_{3}$ (Fig. 4.b) produces bounded position (Fig. 4.a) response. As exposed in Figs. 4.c-d the wrench components that would have led to a violation of these constraints are propagated to the mobile base, thereby generating suitable reactive motions. In practice, since the bounds are tight, most of the applied wrench is directly propagated to the mobile base. In the following experiments, we set $\lambda=\mu=100$ as the robot is moving far from its joint limits.

2) Experiment 2. Contact impedance control with torque limits: The following experiment is focusing on the generation of compliant reactive motions following a desired dynamics, in response to physical interactions between the robot and an external operator. A set of external forces is applied to the second link of TOMM's left arm. This results in an interaction wrench ${ }^{0} \mathbf{W}_{\zeta_{2}}$ located in a centroid $\zeta_{2}$ on the second link of the robot (c.f. Fig. 5.c). The QP solver generates a set of control torques (c.f. Fig. 5.b) that aims to provide the considered centroid with the desired reactive compliant behavior while respecting a set of predefined robot constraints. The desired dynamic parameters of the centroid are the following: 


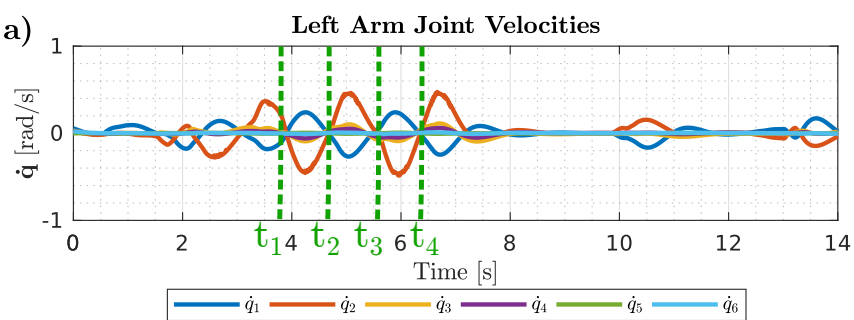

b)

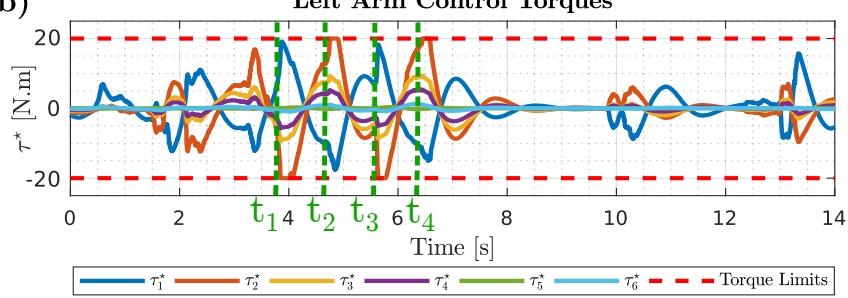

c)

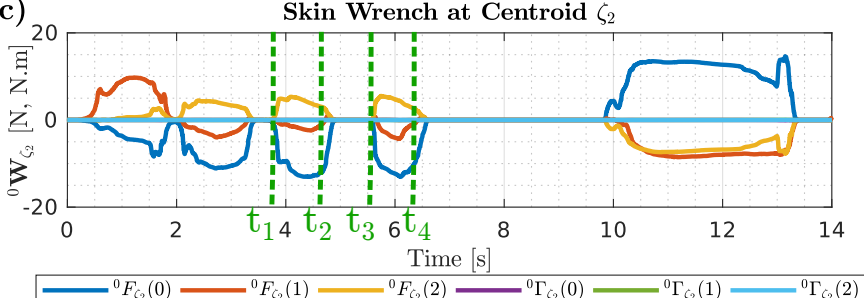

d)

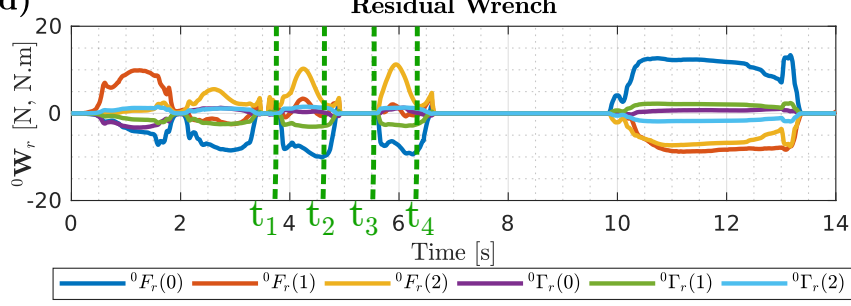

e)

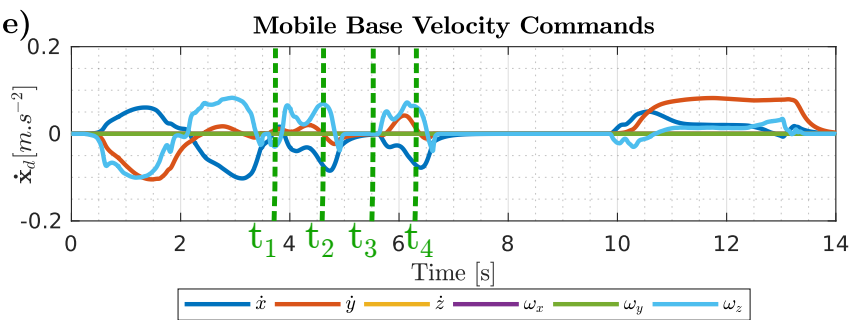

Fig. 5: Contact impedance control

$\boldsymbol{\Lambda}_{d_{\zeta_{3}}}=2.5 \cdot \mathbf{I}_{6 \times 6}, \mathbf{D}_{d_{\zeta_{3}}}=3 \cdot \mathbf{I}_{6 \times 6}$ and $\mathbf{K}_{d_{\zeta_{3}}}=50 \cdot \mathbf{I}_{6 \times 6}$. The mobile base virtual mass is set to $m=25 \mathrm{~kg}$ and its virtual inertia to $I=1.0 \mathrm{~kg} . \mathrm{m}^{2}$. We notice in Fig. 5.a that the joint velocities converge to zero, (e.g. for $10 \leq t \leq 12$ ) while the force is still applied to the robot arm (c.f. Fig. 5.c). This is the expected behavior from a mass-spring damper system with a high stiffness value. Notice on Fig.5.b, the torque saturations occurring at $t_{1}, t_{2}, t_{3}$ and $t_{4}$. These saturations are the result of the torque constraints defined in the QP. In our case, the torque limits are set to $\pm 20 N$.m for each joint. As the torque reaches its limit, the considered robot limb cannot entirely propagate the external forces. The resulting wrench residual (Fig.6.d) must therefore be propagated to the mobile base as shown in Fig.6.e.
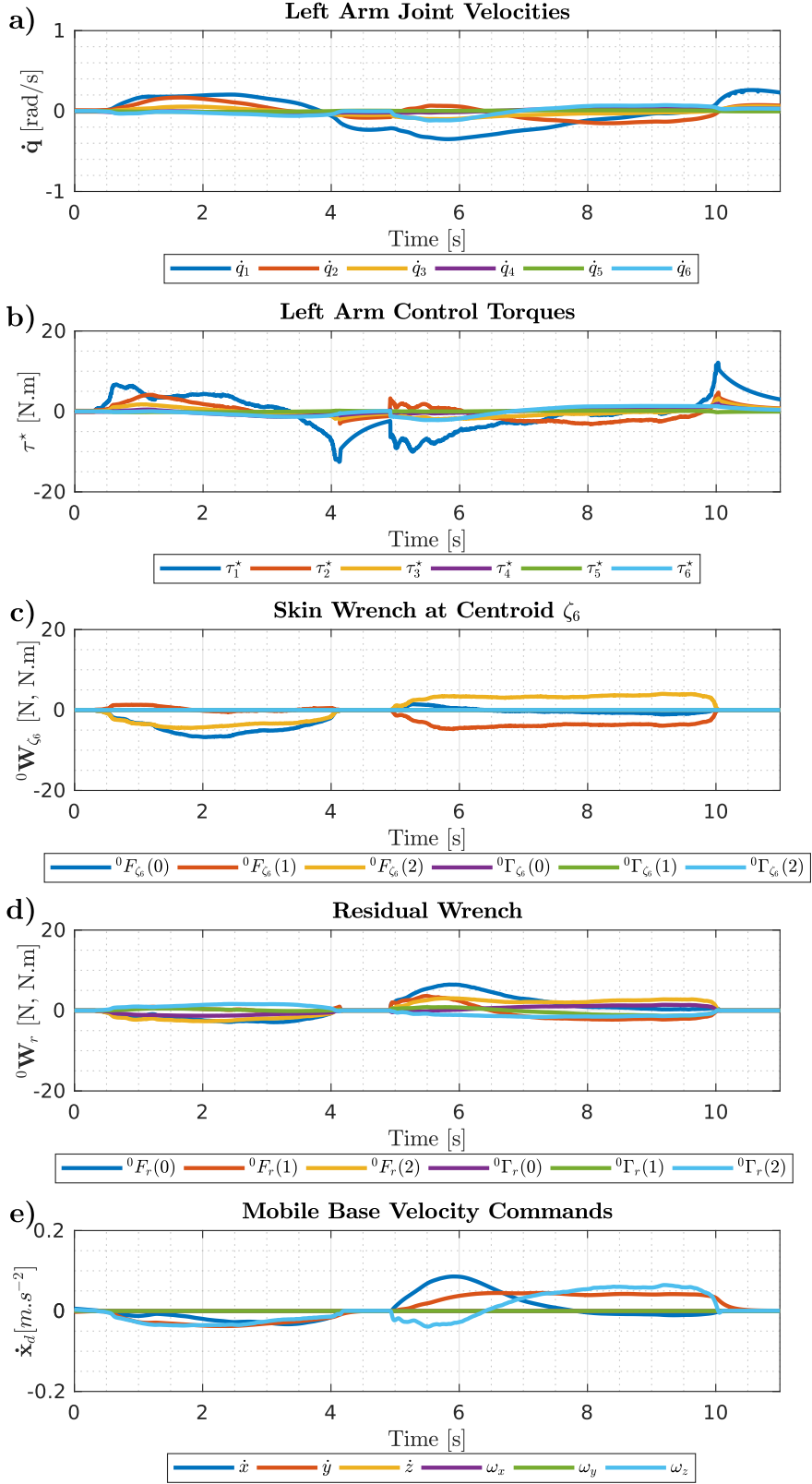

Fig. 6: Fully propagated interactions

3) Experiment 3. Fully propagated interactions: In this experiment, a set of forces is applied by an external operator, to the robot end-effector, thereby resulting in a wrench ${ }^{0} \mathbf{W}_{\boldsymbol{\zeta}_{6}}$ located in the centroid $\boldsymbol{\zeta}_{6}$ (c.f. Fig. 6.c). The desired centroid dynamic parameters are set to the following values: $\boldsymbol{\Lambda}_{d_{\zeta_{3}}}=2.5 \cdot \mathbf{I}_{6 \times 6}, \mathbf{D}_{d_{\zeta_{3}}}=3 \cdot \mathbf{I}_{6 \times 6}$ and $\mathbf{K}_{d_{\zeta_{3}}}=5 \cdot \mathbf{I}_{6 \times 6}$. The mobile base virtual mass is set to $m=20 \mathrm{~kg}$ and its virtual inertia to $I=1.0 \mathrm{~kg} \cdot \mathrm{m}^{2}$. The objective of this experiment is to demonstrate that a force which is properly compensated by a given limb ${ }^{9}$, does not generate any significant residual onto its parent (in our case, on the mobile base). The experimental results, depicted in Fig. 6, show that this is indeed the case: the residual wrench - resp. mobile base motions - are negligible on the considered time frame, as shown in Fig.6.d-e.

${ }^{9}$ Hence the choice of the end-effector for this experiment. 


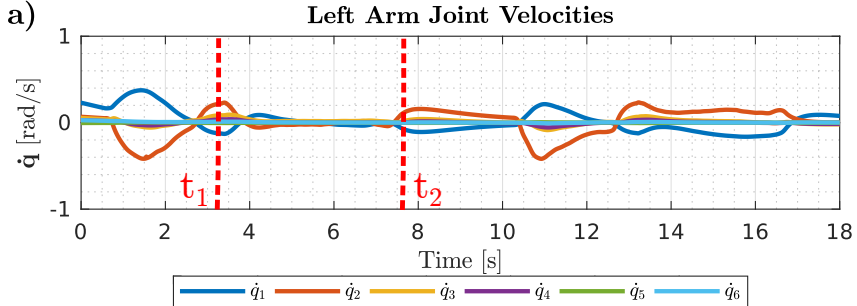

b)

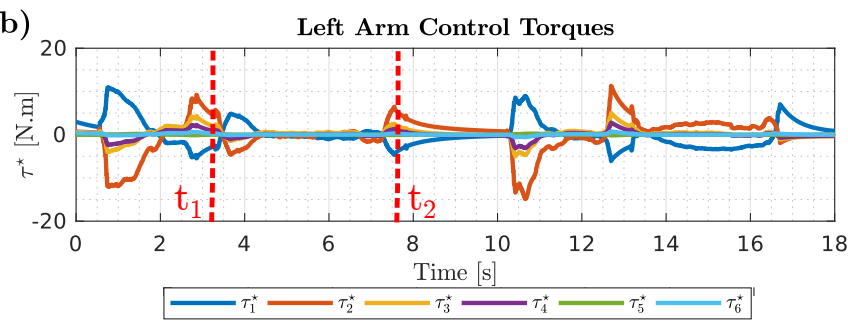

c)

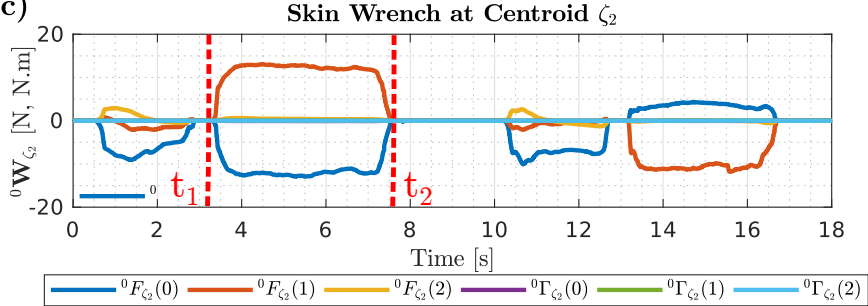

d)
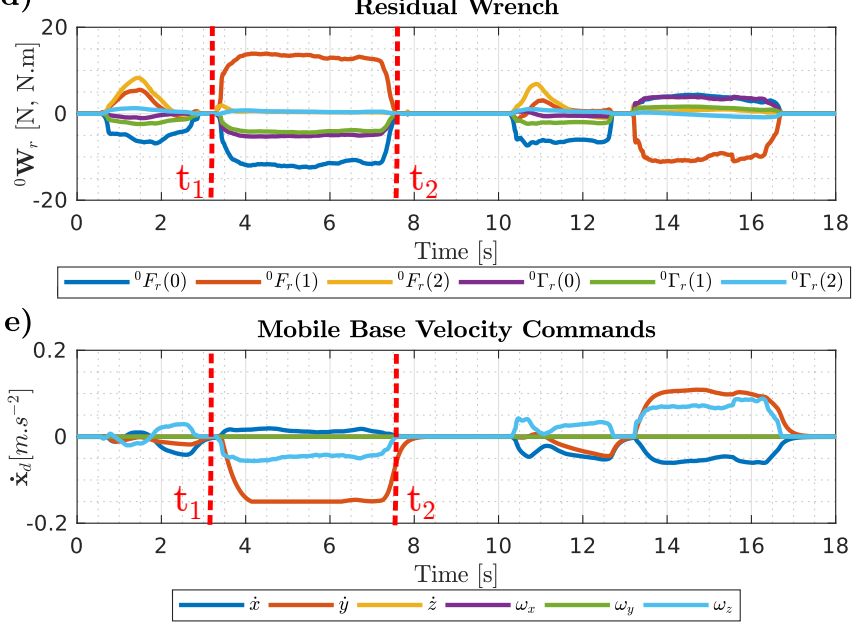

Fig. 7: Nullspace interactions

4) Experiment 4. Nullspace interactions: In this experiment, a force is applied close to the nullspace of the second link, as depicted in Fig. 8. The desired dynamic parameters are the same as those of the previous experiment: $\boldsymbol{\Lambda}_{d_{\zeta_{3}}}=$ $2.5 \cdot \mathbf{I}_{6 \times 6}, \mathbf{D}_{d_{\zeta_{3}}}=3 \cdot \mathbf{I}_{6 \times 6}$ and $\mathbf{K}_{d_{\zeta_{3}}}=5 \cdot \mathbf{I}_{6 \times 6}$. The mobile base virtual mass is here also set to $m=20 \mathrm{~kg}$ and its virtual inertia to $I=1.0 \mathrm{~kg} \cdot \mathrm{m}^{2}$. On a theoretical point of view, since the considered limb cannot generate any compliant reactive movement in the direction of the applied force, this force should therefore be entirely propagated to the mobile base. The experiment results show this behavior for $t_{1} \leq t \leq t_{2}$ we observe in Fig.7.c-e that the applied wrench is entirely transmitted to the mobile base since the arm cannot generate suitable accelerations in the direction of the applied force. The joint velocities and torques (Fig.7.a-b) are moreover negligible

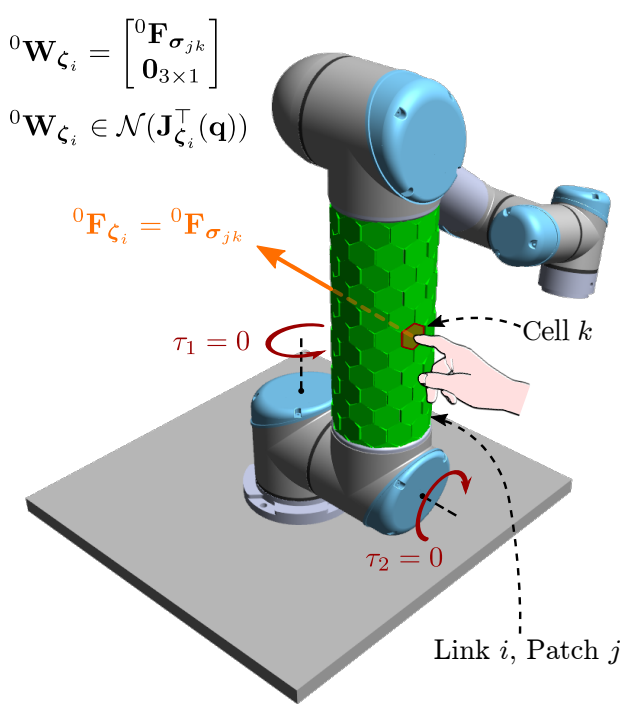

Fig. 8: Single-contact nullspace interaction with the second rigid link of a UR5 robot. Since the wrench ${ }^{0} \mathbf{W}_{\boldsymbol{\zeta}_{i}}$ lies in the nullspace $\mathcal{N}\left(\mathbf{J}_{\boldsymbol{\zeta}_{i}}^{\top}(\mathbf{q})\right)$ of the corresponding contact Jacobian transpose, it cannot produce any torque on the robot kinematic chain, and must therefore be propagated to a parent limb.

compared to the ones obtained in the previous experiments ${ }^{10}$. Notice on Fig.7.e that for $t \geq t_{2}$, the mobile base velocity command is not immediately set to zero, even if the external wrench is no longer applied to the robot. This is a consequence of the mobile base virtual dynamics.

5) Experiment 5. Computational Efficiency: During this experiment, we focus on the evolution of our controller's computational complexity as a function of the number of activated skin cells. For both practical and safety reasons, the robot must remain motionless during the whole process. Therefore although the control torque $\tau^{\star}$ is still computed for timing evaluation purpose, we rather control the real robot with a simple gravity compensation controller $\boldsymbol{\tau}=\mathbf{g}(\mathbf{q})$, resulting in $\ddot{\mathbf{q}}=\mathbf{0}_{n \times 1}$. We then proceed by gradually covering the robot's artificial skin with a set of opaque screens, in order to activate as many proximity sensors as possible. Since the skin's proximity feedback is automatically mapped to a virtual force [22], it is therefore naturally integrated into our controller. The experiment results are shown in Fig. 9aand Fig. 9b. We can observe that the global execution time of the proposed algorithm - in Fig. 9a- fully comply with the real-time constraint of our control loop $(\delta t=8 \mathrm{~ms}$ in dashed red). The results moreover suggest that the influence of the number of activated cells on the computation time of the loop is negligible. Unlike our previous work, where a QP had to be solved for every activated skin cell, the proposed control method now only requires a single QP to be solved for each limb and for each time iteration of the control loop. In the worst case, for TOMM, it is therefore only two QP that will have to be solved at each control iteration ${ }^{11}$. Finally, since each

\footnotetext{
${ }^{10}$ Joint velocities and torques are nonetheless not null, since the external forces are not applied "exactly" to the nullspace of the robot.

${ }^{11}$ The mobile base does not requires the resolution of any QP as it does not have any parent limb.
} 


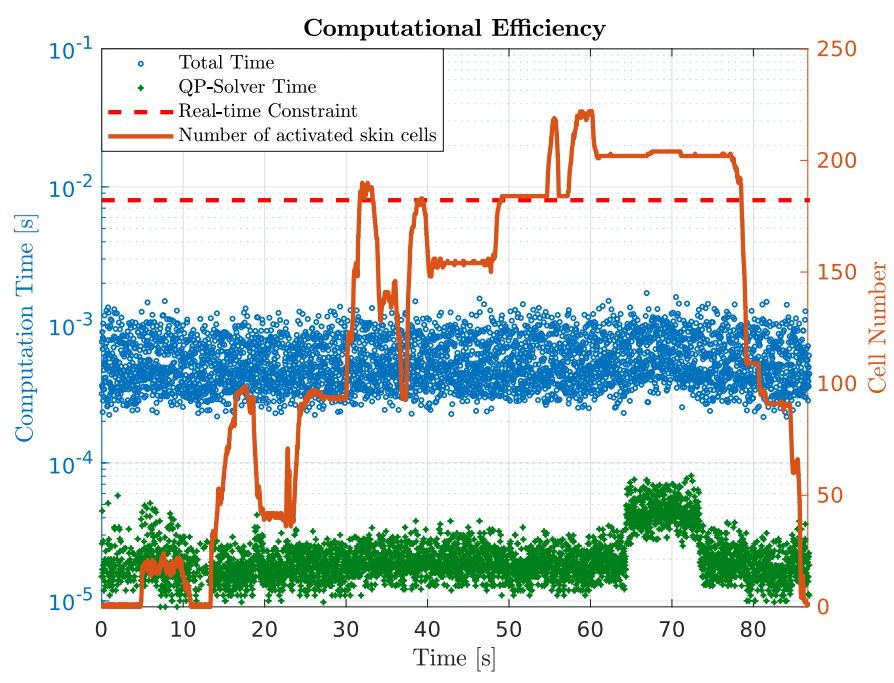

(a) Proposed method

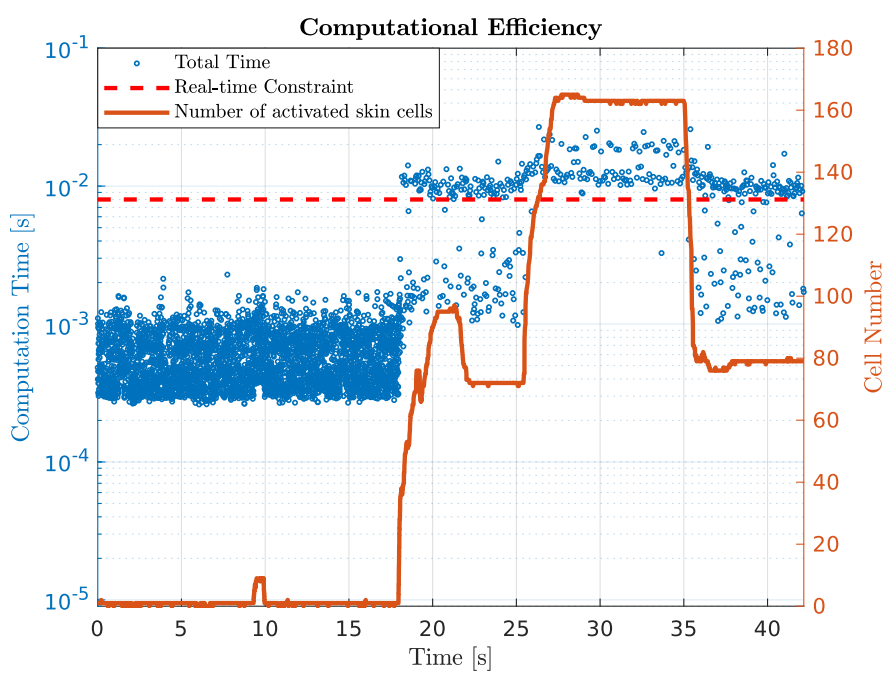

(b) Old framework

Fig. 9: (a): Computational Efficiency of the proposed algorithm. In green the time $t_{q p}$ required to solve the QP (on a semi-log scale). In blue on the same scale, the time $t_{\text {tot }}$ necessary to solve the entire control problem (i.e. acquire skin data, and solve the QP). The real-time loop constraint $t_{\text {loop }}=8 \mathrm{~ms}$ is showed in dashed red. Finally the number of activated cells, sending force and position data is showed in Orange. (b): Computational Efficiency of the Algorithm proposed in our previous work [48]. The time $t_{q p}$ necessary to solve each QP is here not relevant due to the cell-wise formulation of the problem. We notice that the computational complexity increases with the number of activated cells and rapidly violates the real-time constraint.

robot limb is associated with a specific thread, these QP can be solved in parallel which further increases the computational efficiency.

\section{CONClusion}

In this paper, we address the issue of tactile-based wholebody compliance with force propagation for wheeled mobile manipulators. Using the tactile feedback of an artificial skin, we propose a new control paradigm, based on the reactive acceleration error between each of the robot's rigid links, and a corresponding set of virtual mass-spring-damper systems, moving with a predefined compliant dynamics. The use of Quadratic programming makes it possible to determine a set of proper control torques, allowing track as closely as possible the desired reactive behavior while intrinsically complying with the robot physical constraints. In addition to its intuitiveness, formulating the controller in Cartesian space, allows us to conveniently capture the dynamic residuals between the desired and the actual reactive motions of each limb, and to propagate them onto a parent kinematic chain. In this manner, the robot remains compliant under external tactile perturbations, even when the arms reach kinematic or dynamic constraints. Our artificial skin here plays a major role since it provides informations on the magnitude, direction and localization of tactile interactions. The experiments performed on a dual arm mobile manipulator demonstrate the reliability and efficiency of our method as well as its applicability to human-robot interaction scenarios. We provide a video ${ }^{12}$ to illustrate the robot behaviors described in this paper. Future works will mainly consist in extending the developed control paradigm to floating-based robots such as humanoid robots. Further exploitation of the artificial skin multimodality, especially through acceleration and temperature signals, will also be considered.

\section{REFERENCES}

[1] S. Schaal, "The new robotics towards human-centered machines," HFSP journal, vol. 1, no. 2, pp. 115-126, 2007.

[2] P. Lin, K. Abney, and G. Bekey, "Robot ethics: Mapping the issues for a mechanized world," Artificial Intelligence, vol. 175, no. 5, pp. 942-949, 2011.

[3] M. M. de Graaf and S. B. Allouch, "Anticipating our future robot society: The evaluation of future robot applications from a user's perspective," in Robot and Human Interactive Communication (RO-MAN), 2016 25th IEEE International Symposium on, pp. 755-762, IEEE, 2016.

[4] A. Dietrich, K. Bussmann, F. Petit, P. Kotyczka, C. Ott, B. Lohmann, and A. Albu-Schäffer, "Whole-body impedance control of wheeled mobile manipulators," Autonomous Robots, pp. 1-13, 2015.

[5] R. Alami, A. Albu-Schäffer, A. Bicchi, R. Bischoff, R. Chatila, A. De Luca, A. De Santis, G. Giralt, J. Guiochet, G. Hirzinger, et al., "Safe and dependable physical human-robot interaction in anthropic domains: State of the art and challenges," in Intelligent Robots and Systems (IROS), 2006 IEEE/RSJ International Conference on, pp. 116, IEEE, 2006.

[6] N. Hogan, "Impedance control: An approach to manipulation: Part itheory, part ii-implementation, part iii-applications.," Journal of Dynamic Systems, Measurement, and Control, vol. 107, pp. 1-24, 1985.

[7] O. Khatib, "A unified approach for motion and force control of robot manipulators: The operational space formulation," Robotics and Automation, IEEE Journal of, vol. 3, no. 1, pp. 43-53, 1987.

[8] H.-O. Lim and K. Tanie, "Human safety mechanisms of human-friendly robots: passive viscoelastic trunk and passively movable base," The International Journal of Robotics Research, vol. 19, no. 4, pp. 307-335, 2000.

[9] A. Bicchi, S. L. Rizzini, and G. Tonietti, "Compliant design for intrinsic safety: General issues and preliminary design," in Intelligent Robots and Systems, 2001. Proceedings. 2001 IEEE/RSJ International Conference on, vol. 4, pp. 1864-1869, IEEE, 2001.

[10] $\bar{M}$. Zinn, B. Roth, O. Khatib, and J. K. Salisbury, "A new actuation approach for human friendly robot design," The international journal of robotics research, vol. 23, no. 4-5, pp. 379-398, 2004.

${ }^{12} \mathrm{https} / / /$ youtu.be/teKbiiSN2K8 
[11] S.-H. Hyon, J. G. Hale, and G. Cheng, "Full-body compliant humanhumanoid interaction: balancing in the presence of unknown external forces," IEEE Transactions on Robotics, vol. 23, no. 5, pp. 884-898, 2007.

[12] T. Sugaiwa, H. Iwata, and S. Sugano, "Shock absorbing skin design for human-symbiotic robot at the worst case collision," in Humanoid Robots, 2008. Humanoids 2008. 8th IEEE-RAS International Conference on, pp. 481-486, IEEE, 2008.

[13] O. Eiberger, S. Haddadin, M. Weis, A. Albu-Schäffer, and G. Hirzinger, "On joint design with intrinsic variable compliance: Derivation of the dlr qa-joint," in Robotics and Automation (ICRA), 2010 IEEE International Conference on, pp. 1687-1694, IEEE, 2010.

[14] A. Albu-Schäffer, O. Eiberger, M. Fuchs, M. Grebenstein, S. Haddadin, C. Ott, A. Stemmer, T. Wimböck, S. Wolf, C. Borst, et al., "Anthropomorphic soft robotics-from torque control to variable intrinsic compliance," in Robotics research, pp. 185-207, Springer, 2011.

[15] J. De Schutter and H. Van Brussel, "Compliant robot motion i. a formalism for specifying compliant motion tasks," The International Journal of Robotics Research, vol. 7, no. 4, pp. 3-17, 1988.

[16] J. De Schutter and H. Van Brussel, "Compliant robot motion ii. a control approach based on external control loops," The International Journal of Robotics Research, vol. 7, no. 4, pp. 18-33, 1988

[17] M. G. Carmichael, D. Liu, and K. J. Waldron, "A framework for singularity-robust manipulator control during physical human-robot interaction," The International Journal of Robotics Research, vol. 36, no. 57, pp. 861-876, 2017.

[18] X. Li, Y. Pan, G. Chen, and H. Yu, "Multi-modal control scheme for rehabilitation robotic exoskeletons," The International Journal of Robotics Research, vol. 36, no. 5-7, pp. 759-777, 2017.

[19] M. Geravand, W. Rampeltshammer, and A. Peer, "Control of mobility assistive robot for human fall prevention," in Rehabilitation Robotics (ICORR), 2015 IEEE International Conference on, pp. 882-887, IEEE, 2015.

[20] G. Tonietti, R. Schiavi, and A. Bicchi, "Design and control of a variable stiffness actuator for safe and fast physical human/robot interaction," in Robotics and Automation (ICRA), 2005 IEEE International Conference on, pp. 526-531, IEEE, 2005.

[21] A. Albu-Schaffer, O. Eiberger, M. Grebenstein, S. Haddadin, C. Ott, T. Wimbock, S. Wolf, and G. Hirzinger, "Soft robotics," IEEE Robotics \& Automation Magazine, vol. 15, no. 3, 2008.

[22] E. Dean-Leon, F. Bergner, K. Ramirez-Amaro, and G. Cheng, "From multi-modal tactile signals to a compliant control," in Humanoid Robots (Humanoids), 2016 IEEE-RAS 16th International Conference on, pp. 892-898, IEEE, 2016.

[23] E. Lutscher, M. Lawitzky, G. Cheng, and S. Hirche, "A control strategy for operating unknown constrained mechanisms," in Robotics and Automation (ICRA), 2010 IEEE International Conference on, pp. 819824, IEEE, 2010.

[24] R. Dwiputra and G. K. Kraetzschmar, "Haptic interface for domestic service robot," Journal of Automation and Control Engineering Vol, vol. 2, no. 3, 2014.

[25] F. Ferland, D. Létourneau, A. Aumont, J. Frémy, M.-A. Legault, M. Lauria, and F. Michaud, "Natural interaction design of a humanoid robot," Journal of Human-Robot Interaction, vol. 1, no. 2, pp. 118-134, 2012.

[26] F. Ferland, A. Aumont, D. Létourneau, and F. Michaud, "Taking your robot for a walk: Force-guiding a mobile robot using compliant arms," in Proceedings of the 8th ACM/IEEE international conference on Human-robot interaction, pp. 309-316, IEEE Press, 2013.

[27] L. Sentis, J. Petersen, and R. Philippsen, "Implementation and stability analysis of prioritized whole-body compliant controllers on a wheeled humanoid robot in uneven terrains," Autonomous Robots, vol. 35, no. 4, pp. 301-319, 2013.

[28] A. Dietrich, Whole-body impedance control of wheeled humanoid robots, vol. 116. Springer, 2016.

[29] Y. Wang, C. Smith, Y. Karayiannidis, and P. gren, "Whole body control of a dual-arm mobile robot using a virtual kinematic chain," International Journal of Humanoid Robotics, vol. 13, no. 1, 2016.

[30] Y. Hirata, "Coordinated transportation of a single object by omnidirectional mobile robots with body force sensor," Journal of Robotics and Mechatronics, pp. 242-248, 2000.

[31] K. Kosuge, T. Hayashi, Y. Hirata, and R. Tobiyama, "Dance partner robot-ms dancer," in Intelligent Robots and Systems (IROS), 2003 IEEE/RSJ International Conference on, vol. 4, pp. 3459-3464, IEEE, 2003.
[32] T. Takeda, Y. Hirata, and K. Kosuge, "Dance step estimation method based on hmm for dance partner robot," IEEE Transactions on Industrial Electronics, vol. 54, no. 2, pp. 699-706, 2007.

[33] Z. Liu, Y. Koike, T. Takeda, Y. Hirata, K. Chen, and K. Kosuge, "Development of a passive type dance partner robot," in Advanced Intelligent Mechatronics, 2008. AIM 2008. IEEE/ASME International Conference on, pp. 1070-1075, IEEE, 2008.

[34] K. S. Kim, A. S. Kwok, G. C. Thomas, and L. Sentis, "Fully omnidirectional compliance in mobile robots via drive-torque sensor feedback," in Intelligent Robots and Systems (IROS), 2014 IEEE/RSJ International Conference on, pp. 4757-4763, IEEE, 2014.

[35] K. S. Kim, T. Llado, and L. Sentis, "Full-body collision detection and reaction with omnidirectional mobile platforms: a step towards safe human-robot interaction," Autonomous Robots, vol. 40, no. 2, pp. 325$341,2016$.

[36] J. Frémy, F. Ferland, M. Lauria, and F. Michaud, "Force-guidance of a compliant omnidirectional non-holonomic platform," Robotics and Autonomous Systems, vol. 62, no. 4, pp. 579-590, 2014.

[37] D. F. P. Granados, J. Kinugawa, Y. Hirata, and K. Kosuge, "Guiding human motions in physical human-robot interaction through com motion control of a dance teaching robot," in Humanoid Robots (Humanoids), 2016 IEEE-RAS 16th International Conference on, pp. 279-285, IEEE, 2016.

[38] T. L. Chen and C. C. Kemp, "Lead me by the hand: Evaluation of a direct physical interface for nursing assistant robots," in Proceedings of the 5th ACM/IEEE international conference on Human-robot interaction, pp. 367-374, IEEE Press, 2010.

[39] T. L. Chen and C. C. Kemp, "A direct physical interface for navigation and positioning of a robotic nursing assistant," Advanced Robotics, vol. 25, no. 5, pp. 605-627, 2011.

[40] T. L. Chen, T. Bhattacharjee, J. L. McKay, J. E. Borinski, M. E. Hackney, L. H. Ting, and C. C. Kemp, "Evaluation by expert dancers of a robot that performs partnered stepping via haptic interaction," PLOS ONE, vol. 10, pp. 1-24, 52015.

[41] K. Nagasaka, Y. Kawanami, S. Shimizu, T. Kito, T. Tsuboi, A. Miyamoto, T. Fukushima, and H. Shimomura, "Whole-body cooperative force control for a two-armed and two-wheeled mobile robot using generalized inverse dynamics and idealized joint units," in Robotics and Automation (ICRA), 2010 IEEE International Conference on, pp. 33773383, IEEE, 2010.

[42] G. Cannata, M. Maggiali, G. Metta, and G. Sandini, "An embedded artificial skin for humanoid robots," in Multisensor Fusion and Integration for Intelligent Systems (MFI), 2008. IEEE International Conference on, pp. 434-438, IEEE, 2008.

[43] B. D. Argall and A. G. Billard, "A survey of tactile human-robot interactions," Robotics and autonomous systems, vol. 58 , no. 10, pp. 1159 $1176,2010$.

[44] A. Kheddar and A. Billard, "A tactile matrix for whole-body humanoid haptic sensing and safe interaction," in Robotics and Biomimetics (ROBIO), 2011 IEEE International Conference on, pp. 1433-1438, IEEE, 2011.

[45] P. Mittendorfer and G. Cheng, "Humanoid multimodal tactile-sensing modules," Robotics, IEEE Transactions on, vol. 27, no. 3, pp. 401-410, 2011.

[46] P. Mittendorfer, From a Multi-modal Intelligent Cell to a Self-organizing Robotic Skin-Realizing Self and Enriching Robot Tactile Interaction. $\mathrm{PhD}$ thesis, Technische Universität München, 2015.

[47] E. Dean-Leon, B. Pierce, F. Bergner, P. Mittendorfer, K. RamirezAmaro, W. Burger, and G. Cheng, "Tomm: Tactile omnidirectional mobile manipulator," in Robotics and Automation (ICRA), 2017 IEEE International Conference on, pp. 2441-2447, IEEE, 2017.

[48] Q. Leboutet, E. Dean-León, and G. Cheng, "Tactile-based compliance with hierarchical force propagation for omnidirectional mobile manipulators," in Humanoid Robots (Humanoids), 2016 IEEE-RAS 16th International Conference on, pp. 926-931, IEEE, 2016.

[49] M. W. Spong and M. Vidyasagar, Robot dynamics and control. John Wiley \& Sons, 2008.

[50] E. Magrini, F. Flacco, and A. De Luca, "Control of generalized contact motion and force in physical human-robot interaction," in Robotics and Automation (ICRA), 2015 IEEE International Conference on, pp. 22982304, IEEE, 2015.

[51] E. Magrini and A. De Luca, "Human-robot coexistence and contact handling with redundant robots," in Intelligent Robots and Systems, 2017 IEEE/RSJ International Conference on, pp. 4611-4617, IEEE, 2017. 
[52] K. S. Kim and L. Sentis, "Human body part multicontact recognition and detection methodology," in Robotics and Automation (ICRA), 2017 IEEE International Conference on, pp. 1908-1915, IEEE, 2017.

[53] A. De Luca, A. Albu-Schaffer, S. Haddadin, and G. Hirzinger, "Collision detection and safe reaction with the dlr-iii lightweight manipulator arm," in Intelligent Robots and Systems, 2006 IEEE/RSJ International Conference on, pp. 1623-1630, IEEE, 2006.

[54] F. Flacco, A. Paolillo, and A. Kheddar, "Residual-based contacts estimation for humanoid robots," in Humanoid Robots (Humanoids), 2016 IEEE-RAS 16th International Conference on, pp. 409-415, IEEE, 2016.

[55] M. Vukobratović and J. Stepanenko, "On the stability of anthropomorphic systems," Mathematical biosciences, vol. 15, no. 1-2, pp. 1-37, 1972.

[56] S. Kajita, H. Hirukawa, K. Harada, and K. Yokoi, Introduction to humanoid robotics, vol. 101. Springer, 2014.

[57] S. O. Madgwick, A. J. Harrison, P. M. Sharkey, R. Vaidyanathan, and W. S. Harwin, "Measuring motion with kinematically redundant accelerometer arrays: Theory, simulation and implementation," Mechatronics, vol. 23, no. 5, pp. 518-529, 2013.

[58] J. Ferreau, H and al., "qpoases user's manual - version 3.0." http://www.coin-or.org/qpOASES/doc/3.0/manual.pdf, 2014.

[59] E. Lutscher and G. Cheng, "Hierarchical inequality task specification for indirect force controlled robots using quadratic programming," in Intelligent Robots and Systems, 2014 IEEE/RSJ International Conference on, pp. 4722-4727, IEEE, 2014.

[60] P. Mittendorfer and G. Cheng, "Integrating discrete force cells into multimodal artificial skin," in Humanoid Robots (Humanoids), 2012 12th IEEE-RAS International Conference on, pp. 847-852, IEEE, 2012.

[61] N. Mamizuka, M. Sakane, K. Kaneoka, N. Hori, and N. Ochiai, "Kinematic quantitation of the patellar tendon reflex using a tri-axial accelerometer," Journal of Biomechanics, vol. 40, no. 9, pp. 2107-2111, 2007.

[62] J. Ferreau, H, C. Kirches, A. Potschka, G. Bock, H, and M. Diehl, "qpoases: A parametric active-set algorithm for quadratic programming," Mathematical Programming Computation, vol. 6, no. 4, pp. 327-363, 2014.

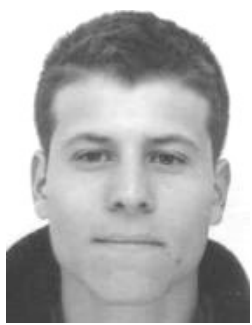

Quentin Leboutet is a Ph.D. candidate in robotics at the Institute for Cognitive Systems of the Technical University of Munich, in Germany since 2016. His current research interests include robot dynamics, parameter estimation, optimal control, sensor fusion, and human-robot interaction/collaboration. He received a M.Eng. degree in Mechatronics from the École National Supérieure de l'Électronique et de ses Applications (ENSEA) and a M.Sc. degree in electrical engineering and computer science from the the Technical University of Munich.

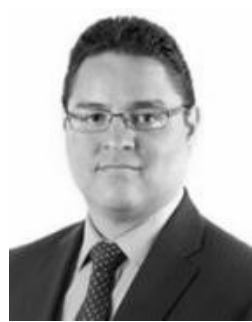

Emmanuel Dean received the M.Sc. and Ph.D. degrees in Mechatronics from the Center for Research and Advanced Studies (CINVESTAV-IPN) in 2003 and 2006. Since 2013 he is a senior researcher at the Chair for Cognitive Systems, Technical University of Munich (TUM). His research interests include robotics, low-level control, and physical humanrobot-interaction/collaboration.

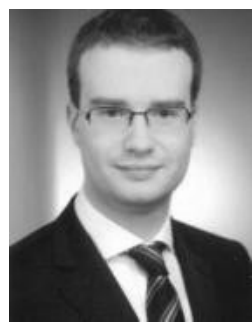

Florian Bergner (M'15) received the B.Sc. and M.Sc. degree in electrical engineering from the Technical University of Munich (TUM), Munich, Germany, in 2011 and 2014, respectively. Since 2014, he has been working toward the Ph.D. degree in electrical engineering at the Chair for Cognitive Systems, TUM. His research interests include multimodal tactile sensing, large-scale robot skin, sensor networks, sensor fusion, neuromorphic engineering, and event-driven signaling and processing.

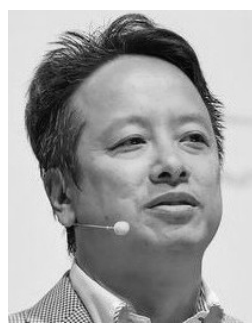

Gordon Cheng Gordon Cheng holds the Chair for Cognitive Systems (2010-), he is Founder and Director of Institute for Cognitive Systems, Faculty of Electrical and Computer Engineering at Technical University of Munich, Munich/Germany. He is also the coordinator of the $\mathrm{CoC}$ for Neuro-Engineering - Center of Competence Neuro-Engineering (2013) in the Department of Electrical and Computer Engineering. Prof. Cheng is speaker of the Elite Master of Science program in Neuroengineering (MSNE) of the Elite Network of Bavaria (2016-). He received a PhD (2011) in Systems Engineering from the Department of Systems Engineering, The Australian National University. Bachelor (1991) and Master (1993) degrees in Computer Science from the University of Wollongong, Australia. He has extensive industrial experiences in consultancy as well as contractual development of large software systems. He was also the Founder/CEO of the company, G.T.I. Computing (1995-2006), a company he founded specialising in networking and transport management systems in Australia. His research interests include, humanoid robotics, cognitive systems, real-time network robot control, brain machine interfaces, bio-mimetic of human vision, computational neuroscience of vision, action understanding, human-robot interaction, active vision, mobile robot navigation and objectoriented software construction. $\mathrm{He}$ is the co-inventor of approximately 20 patents and author of approximately 300 technical publications, proceedings, editorials and book chapters. He has been named IEEE Fellow 2017 for "contributions in humanoid robotic systems and neurorobotics". 